\title{
Sum rules across the unpolarized Compton processes involving generalized polarizabilities and moments of nucleon structure functions
}

\author{
Vadim Lensky, ${ }^{1,2,3}$ Franziska Hagelstein, ${ }^{4}$ Vladimir Pascalutsa, ${ }^{1}$ and Marc Vanderhaeghen ${ }^{1,5}$ \\ ${ }^{1}$ Institut für Kernphysik and Cluster of Excellence PRISMA, \\ Johannes Gutenberg Universität Mainz, D-55099 Mainz, Germany \\ ${ }^{2}$ Institute for Theoretical and Experimental Physics, \\ Bol'shaya Cheremushkinskaya 25, 117218 Moscow, Russia \\ ${ }^{3}$ National Research Nuclear University MEPhI (Moscow Engineering Physics Institute), \\ 115409 Moscow, Russia \\ ${ }^{4}$ Albert Einstein Center for Fundamental Physics, Institute for Theoretical Physics, \\ University of Bern, CH-3012 Bern, Switzerland \\ ${ }^{5}$ Helmholtz Institut Mainz, D-55099 Mainz, Germany
}

(Received 14 December 2017; published 12 April 2018)

\begin{abstract}
We derive two new sum rules for the unpolarized doubly virtual Compton scattering process on a nucleon, which establish novel low- $Q^{2}$ relations involving the nucleon's generalized polarizabilities and moments of the nucleon's unpolarized structure functions $F_{1}\left(x, Q^{2}\right)$ and $F_{2}\left(x, Q^{2}\right)$. These relations facilitate the determination of some structure constants which can only be accessed in off-forward doubly virtual Compton scattering, not experimentally accessible at present. We perform an empirical determination for the proton and compare our results with a next-to-leading-order chiral perturbation theory prediction. We also show how these relations may be useful for a model-independent determination of the low- $Q^{2}$ subtraction function in the Compton amplitude, which enters the two-photon-exchange contribution to the Lamb shift of (muonic) hydrogen. An explicit calculation of the $\Delta(1232)$-resonance contribution to the muonic-hydrogen $2 P-2 S$ Lamb shift yields $-1 \pm 1 \mu \mathrm{eV}$, confirming the previously conjectured smallness of this effect.
\end{abstract}

DOI: 10.1103/PhysRevD.97.074012

\section{INTRODUCTION}

Besides the charge and magnetization distributions in a nucleon, accessed in the elastic lepton-nucleon scattering process, the low-energy nucleon structure is furthermore characterized by its polarizability distributions, which are accessed in Compton scattering (CS) processes with real and virtual photons; see Refs. [1-5] for some reviews.

The CS process is the starting point for deriving sum rules for various electromagnetic structure quantities [6]. For example, the Baldin sum rule for the sum of the dipole polarizabilities [7] and the Gerasimov-Drell-Hearn (GDH) sum rule for the anomalous magnetic moment [8,9] are derived by considering the real Compton scattering (RCS) process. These sum rules all relate a measured low-energy observable to an integral over a photoabsorption cross section on the nucleon and are thus model-independent

Published by the American Physical Society under the terms of the Creative Commons Attribution 4.0 International license. Further distribution of this work must maintain attribution to the author(s) and the published article's title, journal citation, and DOI. Funded by SCOAP ${ }^{3}$. relations. The Burkhardt-Cottingham sum rule [10] has been derived for the forward doubly virtual Compton scattering (VVCS) process, implying that the sum of elastic and inelastic parts of the nucleon's spin structure function $g_{2}$ integrate to zero for arbitrary photon virtualities. Further sum rules involving the spin structure functions were derived by Schwinger [11]. Another important relation by Cottingham [12] connects the unpolarized VVCS with the electromagnetic correction to the proton-neutron mass difference. It can be used to evaluate the electromagnetic part of the proton-neutron mass difference [13,14]. Moreover, further assumption that the high-energy behavior of the VVCS amplitude can be parametrized in terms of Reggeon exchanges leads to separate sum rules for each of the two dipole polarizabilities individually [14].

Recently, we have presented two sum rules $[15,16]$ which extend the GDH, Burkhard-Cottingham, and Schwinger family of sum rules. These new sum rules allow us to connect the moments of the nucleon's low- $Q^{2}$ spin-dependent structure functions $g_{1,2}$, respectively, as measured in inclusive electron scattering $[17,18]$, to lowenergy electromagnetic structure quantities of the nucleon. The latter can be independently obtained in different 
experiments: the nucleon's Pauli radius, two of its four lowest-order spin polarizabilities accessed in RCS [19], and the slopes of two of its four lowest-order generalized polarizabilities (GPs), accessed in the virtual Compton scattering (VCS) process [20-23].

In the present work, we extend such sum-rule relations to the spin-independent VVCS process at low $Q^{2}$. We shall thus derive two new sum rules relating the low- $Q^{2}$ slopes of the second (first) moments of the nucleon's unpolarized structure functions $F_{1}\left(F_{2}\right)$ to structure constants such as the low- $Q^{2}$ slope of the nucleon's electric and magnetic GPs and the quadrupole polarizabilities. We will also show how such relations may be useful for a model-independent determination of the low- $Q^{2}$ "subtraction function" in the forward VVCS amplitude $T_{1}$, which affects prominently the two-photon-exchange (TPE) contribution to the Lamb shift of (muonic) hydrogen.

The paper is organized as follows. In Sec. II, the general formalism for the spin-independent VVCS, i.e., CS with virtual photons in both the initial and final states, and arbitrary kinematics, is introduced. We discuss the Born contributions as well as the low-energy expansions of the non-Born amplitudes. For the latter, we deduce the limits of RCS (Appendix A), VCS (where the incoming photon is virtual and the outgoing photon is real) (Appendix B), and forward VVCS. In Sec. II E, two new sum rules connecting RCS, VCS, and (forward) VVCS quantities are derived, which are verified in Sec. III with a next-to-leading-order baryon chiral perturbation theory (BChPT) calculation of the CS polarizabilities. Furthermore, Sec. II E introduces a new analyticity constraint on the second derivative of the $T_{1}\left(0, Q^{2}\right)$ subtraction function, which is verified and studied in Sec. IV in view of the proton radius puzzle. Empirical and next-to-leading-order (NLO) BChPT predictions for the low-energy coefficients $b_{3,0}, b_{4,1}$, and $b_{19,0}$ are derived based on the newly introduced relations and presented in Secs. III and IV, respectively. In Sec. IV, the effect of the $\Delta(1232)$ excitation on the Lamb shift in muonic hydrogen $(\mu \mathrm{H})$ from TPE is evaluated. The paper finishes with a summary and conclusions (Sec. V).

\section{DOUBLY VIRTUAL COMPTON SCATTERING: SPIN-INDEPENDENT AMPLITUDE}

The main subject of this work is the Compton scattering process shown in Fig. 1, where the photons are, in general, virtual. The spin of the target particle will not play much role in what follows since we will be focusing on the spinindependent observables. Nonetheless, the way the static polarizabilities are defined (in the rest frame of the target), the recoil corrections may bring the dependence on the spin polarizabilities, and hence for those effects, the spin needs to be specified. Keeping in mind the possible applications of this formalism, we take the nucleon as the target particle and hence limit ourselves to the spin- $1 / 2$ case. We

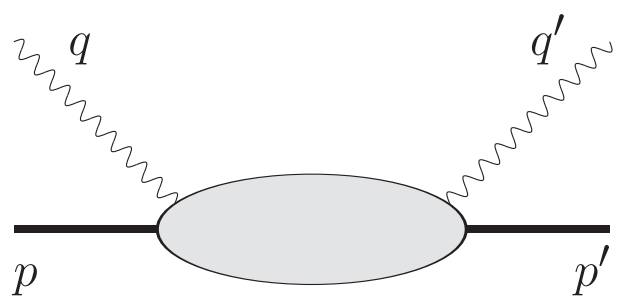

FIG. 1. Diagram of the Compton scattering process showing the 4-momenta of the particles.

therefore consider the doubly virtual Compton scattering process on the nucleon,

$$
\gamma^{*}(q, \lambda)+N(p, s) \rightarrow \gamma^{*}\left(q^{\prime}, \lambda^{\prime}\right)+N\left(p^{\prime}, s^{\prime}\right)
$$

where $\lambda$ and $\lambda^{\prime}$ denote the photon helicities $(0, \pm 1)$ and $s$ and $s^{\prime}$ are the nucleon helicities $( \pm 1 / 2)$.

\section{A. Tensor decomposition}

The VVCS tensor $T^{\mu \nu}$ can be Lorentz decomposed into 18 invariant amplitudes as (in the notation of Ref. [24])

$$
\begin{aligned}
M^{\mu \nu} & =\sum_{i \in J} B_{i}\left(q^{2}, q^{\prime 2}, q \cdot q^{\prime}, q \cdot P\right) T_{i}^{\mu \nu}, \\
J & =\{1, \ldots, 21\} \backslash\{5,15,16\},
\end{aligned}
$$

with $P=\frac{1}{2}\left(p+p^{\prime}\right)$. The 18 independent tensors $T_{i}^{\mu \nu}$ in Eq. (2) are constructed to be gauge invariant [25]. Note that in the most general case one has to use the basis consisting of all 21 tensor amplitudes introduced in Ref. [24] in order to avoid kinematic constraints; however, as long as only the non-Born part of the VVCS amplitude is important (which is the case in the present work, as detailed below), one can use the minimal decomposition of Eq. (2); see Refs. [24,25].

The invariant amplitudes $B_{i}$ depend in general on four kinematic invariants. The incoming (outgoing) photon virtualities are denoted by $q^{2}\left(q^{\prime 2}\right)$, respectively. We also define the usual virtualities $Q^{2}=-q^{2}$ and $Q^{\prime 2}=-q^{\prime 2}$ that are positive for spacelike virtual photons. These two definitions of a photon's virtuality can be used interchangeably, multiplying the appropriate sign factors where needed. Furthermore, the variable $q \cdot q^{\prime}=\left(q^{2}+q^{\prime 2}-t\right) / 2$ is related with the momentum transfer to the nucleon, $t \equiv\left(p^{\prime}-p\right)^{2}$. The crossing symmetric variable $q \cdot P \equiv M \nu$, with $M$ the nucleon mass, can be expressed in terms of the Mandelstam variables $s$ and $u: M \nu \equiv(s-u) / 4$.

As mentioned, we are only interested in the spinindependent case, which is described by five independent tensors,

$\left.M^{\mu \nu}\right|_{\text {spin indep }}=B_{1} T_{1}^{\mu \nu}+B_{2} T_{2}^{\mu \nu}+B_{3} T_{3}^{\mu \nu}+B_{4} T_{4}^{\mu \nu}+B_{19} T_{19}^{\mu \nu}$, 
where the tensors $T_{i}^{\mu \nu}$ are symmetric under exchange of the two virtual photons and are given by

$$
\begin{aligned}
& T_{1}^{\mu \nu}=-q \cdot q^{\prime} g^{\mu \nu}+q^{\prime \mu} q^{\nu} \\
& T_{2}^{\mu \nu}=(2 M \nu)^{2}\left(-g^{\mu \nu}+\frac{q^{\prime \mu} q^{\nu}}{q \cdot q^{\prime}}\right)-4 q \cdot q^{\prime}\left(P^{\mu}-\frac{q \cdot P}{q \cdot q^{\prime}} q^{\prime \mu}\right)\left(P^{\nu}-\frac{q \cdot P}{q \cdot q^{\prime}} q^{\nu}\right), \\
& T_{3}^{\mu \nu}=q^{2} q^{\prime 2} g^{\mu \nu}+q \cdot q^{\prime} q^{\mu} q^{\prime \nu}-q^{2} q^{\prime \mu} q^{\prime \nu}-q^{\prime 2} q^{\mu} q^{\nu}, \\
& T_{4}^{\mu \nu}=(2 M \nu)\left(q^{2}+q^{\prime 2}\right)\left(g^{\mu \nu}-\frac{q^{\prime \mu} q^{\nu}}{q \cdot q^{\prime}}\right)+2\left(P^{\mu}-\frac{q \cdot P}{q \cdot q^{\prime}} q^{\prime \mu}\right)\left(-q^{\prime 2} q^{\nu}+q \cdot q^{\prime} q^{\prime \nu}\right)+2\left(-q^{2} q^{\prime \mu}+q \cdot q^{\prime} q^{\mu}\right)\left(P^{\nu}-\frac{q \cdot P}{q \cdot q^{\prime}} q^{\nu}\right), \\
& T_{19}^{\mu \nu}=4 q^{2} q^{\prime 2}\left(P^{\mu}-\frac{q \cdot P}{q^{2}} q^{\mu}\right)\left(P^{\nu}-\frac{q \cdot P}{q^{\prime 2}} q^{\prime \nu}\right) .
\end{aligned}
$$

The invariant amplitudes $B_{i}$ have definite transformation properties with respect to photon crossing, as well as nucleon crossing combined with charge conjugation [24]. Using the tensors of Eq. (4), the photon crossing symmetry of the whole amplitude $\left(\mu \leftrightarrow \nu, q \leftrightarrow-q^{\prime}\right)$ leads to the following relations for the invariant amplitudes:

$$
\begin{array}{ll}
B_{i}\left(q^{2}, q^{\prime 2}, q \cdot q^{\prime}, q \cdot P\right)=+B_{i}\left(q^{\prime 2}, q^{2}, q \cdot q^{\prime},-q \cdot P\right), & (i=1,2,3,19), \\
B_{i}\left(q^{2}, q^{\prime 2}, q \cdot q^{\prime}, q \cdot P\right)=-B_{i}\left(q^{\prime 2}, q^{2}, q \cdot q^{\prime},-q \cdot P\right), & (i=4) .
\end{array}
$$

Furthermore, nucleon crossing combined with charge conjugation $(P \leftrightarrow-P)$ leads to the relations

$$
\begin{array}{ll}
B_{i}\left(q^{2}, q^{\prime 2}, q \cdot q^{\prime}, q \cdot P\right)=+B_{i}\left(q^{2}, q^{\prime 2}, q \cdot q^{\prime},-q \cdot P\right), & (i=1,2,3,19), \\
B_{i}\left(q^{2}, q^{\prime 2}, q \cdot q^{\prime}, q \cdot P\right)=-B_{i}\left(q^{2}, q^{\prime 2}, q \cdot q^{\prime},-q \cdot P\right), & (i=4) .
\end{array}
$$

\section{B. Born contribution}

An important contribution to the nucleon Compton amplitude at low energies corresponds with a nucleon intermediate state in the blob of Fig. 1, referred to as the Born term. This contribution is, by definition, not affected by structure-dependent constants, such as polarizabilities. The Born term is defined by using the electromagnetic vertex for the transition $\gamma^{*}(q)+N(p) \rightarrow N(p+q)$ given as

$$
\Gamma^{\mu}=F_{D}\left(q^{2}\right) \gamma^{\mu}+F_{P}\left(q^{2}\right) i \sigma^{\mu \nu} \frac{q_{\nu}}{2 M},
$$

with $F_{D}$ and $F_{P}$ the Dirac and Pauli form factors of nucleon $N$, normalized as $F_{D}(0)=e_{N}$ and $F_{P}(0)=\kappa_{N}$, where $e_{N}$ is the charge in units of the proton charge $e\left(e_{N}=0\right.$ for the neutron) and $\kappa_{N}$ is the anomalous magnetic moment in units of the nuclear magneton $e / 2 M ; \sigma^{\mu \nu}=(i / 2)\left[\gamma^{\mu}, \gamma^{\nu}\right]$. With this choice, the Born contribution to the spin-independent VVCS amplitudes is given by

$$
\begin{aligned}
& B_{1}^{\text {Born }}=\frac{1}{4 M^{3}} F_{P}\left(q^{2}\right) F_{P}\left(q^{\prime 2}\right)-\frac{\nu_{B}}{2 M^{2}} \frac{1}{\nu^{2}-\nu_{B}^{2}+i \varepsilon}\left\{G_{M}\left(q^{2}\right) G_{M}\left(q^{\prime 2}\right)-F_{D}\left(q^{2}\right) F_{D}\left(q^{\prime 2}\right)+\frac{q \cdot q^{\prime}}{4 M^{2}} F_{P}\left(q^{2}\right) F_{P}\left(q^{\prime 2}\right)\right\}, \\
& B_{2}^{\text {Born }}=\frac{1}{4 M^{3}} \frac{1}{\nu^{2}-\nu_{B}^{2}+i \varepsilon}\left\{F_{D}\left(q^{2}\right) F_{D}\left(q^{\prime 2}\right)-\frac{q \cdot q^{\prime}}{4 M^{2}} F_{P}\left(q^{2}\right) F_{P}\left(q^{\prime 2}\right)\right\}, \\
& B_{3}^{\text {Born }}=B_{4}^{\text {Born }}=B_{19}^{\text {Born }}=0,
\end{aligned}
$$

where $\nu_{B} \equiv-q \cdot q^{\prime} /(2 M)$, and we introduced the Sachs magnetic form factor, $G_{M}=F_{D}+F_{P}$.

The Born contribution of Eq. (8) can be split into pole and nonpole contributions. The pole contributions (also called elastic contributions) are singular at $\nu=\nu_{B}$. The only nonpole piece in Eq. (8) is obviously the first term, i.e., $B_{1}^{\mathrm{np}}=F_{P}\left(q^{2}\right) F_{P}\left(q^{\prime 2}\right) / 4 M^{3}$. The rest of the Born terms are the pole contributions.

\section{Low-energy expansions}

The non-Born part of the VVCS amplitudes (denoted as $\bar{B}_{i}$ ) can be expanded for small values of $q^{2}, q^{\prime 2}, q \cdot q^{\prime}$, and $\nu$, with the expansion coefficients given by polarizabilities. We use the low-energy expansions (LEXs) in $k=\left\{q, q^{\prime}\right\}$ established in Ref. [24], 


$$
\begin{aligned}
\bar{B}_{i}= & b_{i, 0}+b_{i, 2 a} q \cdot q^{\prime}+b_{i, 2 b}\left(q^{2}+q^{\prime 2}\right) \\
& +b_{i, 2 c}(2 M \nu)^{2}+\mathcal{O}\left(k^{4}\right), \quad(i=1,2,3,19), \\
\bar{B}_{i}= & 2 M \nu\left\{b_{i, 1}+b_{i, 3 a} q \cdot q^{\prime}+b_{i, 3 b}\left(q^{2}+q^{\prime 2}\right)\right. \\
& \left.+b_{i, 3 c}(2 M \nu)^{2}+\mathcal{O}\left(k^{4}\right)\right\}, \quad(i=4),
\end{aligned}
$$

where the parameters $b_{i, x}$ are structure constants. We notice that in order to fully specify the low-energy structure of the spin-independent doubly virtual Compton amplitude one requires two constants at the lowest order $\left(b_{1,0}\right.$ and $\left.b_{2,0}\right)$ and nine additional constants when going to the next order: six coefficients arising from higher-order terms in $\bar{B}_{1}$ and $\bar{B}_{2}$ and the three lowest-order coefficients in the amplitudes $\bar{B}_{3}, \bar{B}_{4}$, and $\bar{B}_{19}$, which are the amplitudes which are accompanied by tensor structures of higher order in $k=\left\{q, q^{\prime}\right\}$.

The RCS process, corresponding with $q^{2}=q^{\prime 2}=0$, allows one to constrain the two lowest-order coefficients in $\bar{B}_{1}$ and $\bar{B}_{2}$ as well as four of the next-order coefficients in $\bar{B}_{1}$ and $\bar{B}_{2}$. We detail the connection between these coefficients and the polarizabilities, accessible through RCS, in Appendix A. These relations are given by (with the fine-structure constant $\alpha_{\mathrm{em}} \equiv e^{2} / 4 \pi \simeq 1 / 137$ ):

$$
\begin{aligned}
b_{1,0}= & \frac{1}{\alpha_{\mathrm{em}}} \beta_{M 1}, \\
b_{1,2 a}= & -\frac{1}{\alpha_{\mathrm{em}}} \frac{1}{6} \beta_{M 2} \\
b_{1,2 c}= & \frac{1}{\alpha_{\mathrm{em}}} \frac{1}{(2 M)^{2}}\left[\beta_{M 1, \nu}+\frac{1}{12}\left(2 \beta_{M 2}-\alpha_{E 2}\right)\right. \\
& \left.+\frac{1}{M}\left(\gamma_{M 1 M 1}+\gamma_{E 1 M 2}\right)\right] \\
b_{2,0}= & -\frac{1}{\alpha_{\mathrm{em}}} \frac{1}{(2 M)^{2}}\left(\alpha_{E 1}+\beta_{M 1}\right), \\
b_{2,2 a}= & \frac{1}{\alpha_{\mathrm{em}}} \frac{1}{(2 M)^{2}}\left[\frac{1}{6}\left(\alpha_{E 2}+\beta_{M 2}\right)\right. \\
& \left.-\frac{1}{M}\left(\gamma_{E 1 M 2}+\gamma_{M 1 E 2}\right)+\frac{1}{2 M^{2}}\left(\alpha_{E 1}+\beta_{M 1}\right)\right] \\
b_{2,2 c}= & -\frac{1}{\alpha_{\mathrm{em}}} \frac{1}{(2 M)^{4}}\left[\alpha_{E 1, \nu}+\beta_{M 1, \nu}+\frac{1}{12}\left(\alpha_{E 2}+\beta_{M 2}\right)\right] .
\end{aligned}
$$

Besides the electric (magnetic) dipole polarizabilities $\alpha_{E 1}\left(\beta_{M 1}\right)$, the above relations involve the corresponding electric (magnetic) dispersive polarizabilities $\alpha_{E 1, \nu}\left(\beta_{M 1, \nu}\right)$ and the electric (magnetic) quadrupole polarizabilities $\alpha_{E 2}$ $\left(\beta_{M 2}\right)$. Furthermore, there are recoil terms (proportional to $1 / M$ relative to the quadrupole polarizability terms), which involve the lowest-order nucleon spin polarizabilities $\gamma_{M 1 M 1}, \gamma_{E 1 M 2}$, and $\gamma_{M 1 E 2}$, as well as recoil terms (proportional to $1 / M^{2}$ ), which involve the scalar polarizabilities $\alpha_{E 1}$ and $\beta_{M 1}$.

In Appendix B, we show that the nonforward VCS process, corresponding with an outgoing real photon, i.e., $q^{\prime 2}=0$, and an initial spacelike virtual photon with virtuality $q^{2}$, provides a second limit for the doubly virtual Compton scattering. Its measurement allows us to constrain two more of the next-order coefficients in $\bar{B}_{1}$ and $\bar{B}_{2}$ as

$$
\begin{aligned}
b_{1,2 b}= & -\frac{1}{\alpha_{\mathrm{em}}}\left\{\beta_{M 1}^{\prime}+\frac{1}{8 M^{2}} \beta_{M 1}\right\}, \\
b_{2,2 b}= & \frac{1}{\alpha_{\mathrm{em}}} \frac{1}{(2 M)^{2}}\left\{\alpha_{E 1}^{\prime}+\beta_{M 1}^{\prime}-\frac{1}{2 M}\left(\delta_{L T}+\gamma_{E 1 E 1}-\gamma_{E 1 M 2}\right)\right. \\
& \left.-\frac{1}{8 M^{2}}\left(\alpha_{E 1}+\beta_{M 1}\right)\right\}
\end{aligned}
$$

which involve the slopes at $Q^{2}=0$ of the magnetic $\left(\beta_{M 1}^{\prime}\right)$ and electric $\left(\alpha_{E 1}^{\prime}\right)$ GPs, defined through Eqs. (B7a) and (B7b). Furthermore, the recoil terms (proportional to $1 / M$ and $1 / M^{2}$ relative to $\beta_{M 1}^{\prime}$ or $\alpha_{E 1}^{\prime}$ ) involve, besides $\alpha_{E 1}$ and $\beta_{M 1}$, also the RCS spin polarizabilities $\gamma_{E 1 E 1}, \gamma_{E 1 M 2}$, as well as the longitudinal-transverse spin polarizability $\delta_{L T}$ at $Q^{2}=0$, which is accessed from a moment of the nucleon spin-dependent structure functions $g_{1}$ and $g_{2}$.

We note that all quantities entering the rhs of Eqs. (10a)-(10f) and Eqs. (11a)-(11b) are observables which are accessed either through the RCS process, VCS process, or forward structure functions.

\section{Forward limit}

Besides the low-energy RCS and VCS processes, we can consider as another limit of the doubly virtual Compton process of Eq. (1) the forward VVCS limit, which corresponds with $q^{\prime}=q$ and $p^{\prime}=p$. Notice that for this process $q^{2}=q^{\prime 2}=q \cdot q^{\prime}=-Q^{2}<0$. The helicity averaged forward VVCS process is described by two invariant amplitudes, denoted by $T_{1}$ and $T_{2}$, which are functions of two kinematic invariants: $Q^{2}$ and $\nu$. Its covariant tensor structure can be written as

$$
\begin{aligned}
& \left.\alpha_{\mathrm{em}} M^{\mu \nu}(\mathrm{VVCS})\right|_{\text {spin indep }} \\
& \equiv\left(g^{\mu \nu}-\frac{q^{\mu} q^{\nu}}{q^{2}}\right) T_{1}\left(\nu, Q^{2}\right) \\
& \quad-\frac{1}{M^{2}}\left(p^{\mu}-\frac{p \cdot q}{q^{2}} q^{\mu}\right)\left(p^{\nu}-\frac{p \cdot q}{q^{2}} q^{\nu}\right) T_{2}\left(\nu, Q^{2}\right),
\end{aligned}
$$

where $\alpha_{\mathrm{em}}$ is conventionally introduced in defining the forward amplitudes $T_{1}$ and $T_{2}$. The optical theorem relates 
the imaginary parts of $T_{1}$ and $T_{2}$ to the two unpolarized structure functions of inclusive electron-nucleon scattering as

$$
\begin{aligned}
\operatorname{Im} T_{1}\left(\nu, Q^{2}\right) & =\frac{e^{2}}{4 M} F_{1}\left(x, Q^{2}\right), \\
\operatorname{Im} T_{2}\left(\nu, Q^{2}\right) & =\frac{e^{2}}{4 \nu} F_{2}\left(x, Q^{2}\right),
\end{aligned}
$$

where $x \equiv Q^{2} / 2 M \nu$ and where $F_{1}$ and $F_{2}$ are the conventionally defined structure functions parametrizing inclusive electron-nucleon scattering. The imaginary parts of the forward scattering amplitudes, Eq. (13), get contributions from both elastic scattering at $\nu=\nu_{B} \equiv Q^{2} /(2 M)$, or equivalently $x=1$, as well as from inelastic processes above the pion production threshold, corresponding with $\nu>\nu_{0} \equiv m_{\pi}+\left(Q^{2}+m_{\pi}^{2}\right) /(2 M)$ with $m_{\pi}$ the pion mass, or equivalently $x<x_{0} \equiv Q^{2} /\left(2 M \nu_{0}\right)$.

Expressing the doubly virtual Compton tensors of Eq. (4) in the forward limit, the VVCS amplitudes $T_{1}$ and $T_{2}$ can be readily expressed in terms of the $B_{i}$ amplitudes of Eq. (3) as

$$
T_{1}\left(\nu, Q^{2}\right)=\alpha_{\mathrm{em}}\left\{Q^{2} B_{1}-4 M^{2} \nu^{2} B_{2}+Q^{4} B_{3}-4 M \nu Q^{2} B_{4}\right\},
$$

$$
T_{2}\left(\nu, Q^{2}\right)=\alpha_{\mathrm{em}} 4 M^{2} Q^{2}\left\{-B_{2}-Q^{2} B_{19}\right\},
$$

where the amplitudes $B_{i}$ also depend on $\nu$ and $Q^{2}$ for forward kinematics.

Using Eq. (8), we can express the Born contributions in the forward limit as

$$
\begin{aligned}
& T_{1}^{\mathrm{Born}}=-\frac{\alpha_{\mathrm{em}}}{M}\left(F_{D}^{2}\left(q^{2}\right)+\frac{\nu_{B}^{2}}{\nu^{2}-\nu_{B}^{2}+i \varepsilon} G_{M}^{2}\left(q^{2}\right)\right), \\
& T_{2}^{\mathrm{Born}}=-\frac{\alpha_{\mathrm{em}}}{M} \frac{Q^{2}}{\nu^{2}-\nu_{B}^{2}+i \varepsilon}\left(F_{D}^{2}\left(q^{2}\right)+\frac{Q^{2}}{4 M^{2}} F_{P}^{2}\left(q^{2}\right)\right),
\end{aligned}
$$

and the corresponding pole parts as

$$
\begin{aligned}
\operatorname{Re} T_{1}^{\mathrm{pole}} & =-\frac{\alpha_{\mathrm{em}}}{M} \frac{\nu_{B}^{2}}{\nu^{2}-\nu_{B}^{2}} G_{M}^{2}\left(q^{2}\right) \\
\operatorname{Re} T_{2}^{\mathrm{pole}} & =-\frac{\alpha_{\mathrm{em}}}{M} \frac{Q^{2}}{\nu^{2}-\nu_{B}^{2}}\left(F_{D}^{2}\left(q^{2}\right)+\frac{Q^{2}}{4 M^{2}} F_{P}^{2}\left(q^{2}\right)\right) .
\end{aligned}
$$

Using the LEXs of the non-Born amplitudes $\bar{B}_{i}$, given in Eqs. (9a) and (9b), we can obtain from Eqs. (14) and (15) LEXs for the non-Born parts $\bar{T}_{1,2}$ of the amplitudes $T_{1,2}$. Up to fourth order in $k=\{\nu, Q\}$, these LEXs are given by

$$
\begin{aligned}
\bar{T}_{1}\left(\nu, Q^{2}\right)= & \alpha_{\mathrm{em}}\left\{Q^{2} b_{1,0}-4 M^{2} \nu^{2} b_{2,0}\right. \\
& +Q^{4}\left[-b_{1,2 a}-2 b_{1,2 b}+b_{3,0}\right]-(2 M \nu)^{4} b_{2,2 c} \\
& \left.+(2 M \nu)^{2} Q^{2}\left[b_{1,2 c}+b_{2,2 a}+2 b_{2,2 b}-2 b_{4,1}\right]\right\} \\
& +\mathcal{O}\left(k^{6}\right),
\end{aligned}
$$

$$
\begin{aligned}
\bar{T}_{2}\left(\nu, Q^{2}\right)= & -\alpha_{\mathrm{em}} 4 M^{2} Q^{2}\left\{b_{2,0}+Q^{2}\left[-b_{2,2 a}-2 b_{2,2 b}+b_{19,0}\right]\right. \\
& \left.+(2 M \nu)^{2} b_{2,2 c}\right\}+\mathcal{O}\left(k^{6}\right) .
\end{aligned}
$$

Besides the low-energy coefficients constrained from RCS and VCS, as given in Eqs. (10a)-(10f) and Eqs. (11a) and (11b), the knowledge of the amplitudes $\bar{T}_{1}$ and $\bar{T}_{2}$ to fourth order requires in addition the knowledge of the constants $b_{3,0}, b_{4,1}$, and $b_{19,0}$, which we will discuss in the next section.

In the following, we will also be interested in the amplitude $\bar{T}_{1}$ at zero energy $(\nu=0)$, which plays the role of a subtraction function in a dispersive framework for the VVCS amplitude. From Eq. (18), we see that its LEX can be expressed as

$$
\begin{aligned}
\bar{T}_{1}\left(0, Q^{2}\right)= & \alpha_{\mathrm{em}}\left\{Q^{2} b_{1,0}+Q^{4}\left[-b_{1,2 a}-2 b_{1,2 b}+b_{3,0}\right]\right\} \\
& +\mathcal{O}\left(Q^{6}\right) .
\end{aligned}
$$

\section{E. Sum rules}

Using the RCS constraints of Eqs. (10a)-(10f) and the VCS constraints of Eqs. (11a) and (11b) on the low-energy coefficients, we can express the spin-independent VVCS amplitudes of Eqs. (18) and (19) including all terms up to fourth order in either $Q$ or $\nu$ as

$$
\begin{aligned}
\bar{T}_{1}\left(\nu, Q^{2}\right)= & Q^{2} \beta_{M 1}+\nu^{2}\left(\alpha_{E 1}+\beta_{M 1}\right)+\nu^{4}\left[\alpha_{E 1, \nu}+\beta_{M 1, \nu}+\frac{1}{12}\left(\alpha_{E 2}+\beta_{M 2}\right)\right] \\
& +Q^{2} \nu^{2}\left[\beta_{M 1, \nu}+\frac{1}{12}\left(4 \beta_{M 2}+\alpha_{E 2}\right)+2\left(\alpha_{E 1}^{\prime}+\beta_{M 1}^{\prime}\right)-2 \alpha_{\mathrm{em}}(2 M)^{2} b_{4,1}\right. \\
& \left.+\frac{1}{M}\left(-\delta_{L T}+\gamma_{M 1 M 1}-\gamma_{E 1 E 1}-\gamma_{M 1 E 2}+\gamma_{E 1 M 2}\right)+\frac{1}{(2 M)^{2}}\left(\alpha_{E 1}+\beta_{M 1}\right)\right] \\
& +Q^{4}\left[\frac{1}{6} \beta_{M 2}+2 \beta_{M 1}^{\prime}+\alpha_{\mathrm{em}} b_{3,0}+\frac{1}{(2 M)^{2}} \beta_{M 1}\right]+\mathcal{O}\left(k^{6}\right),
\end{aligned}
$$




$$
\begin{aligned}
\bar{T}_{2}\left(\nu, Q^{2}\right)= & Q^{2}\left(\alpha_{E 1}+\beta_{M 1}\right)+Q^{2} \nu^{2}\left[\alpha_{E 1, \nu}+\beta_{M 1, \nu}+\frac{1}{12}\left(\alpha_{E 2}+\beta_{M 2}\right)\right]+Q^{4}\left[\frac{1}{6}\left(\alpha_{E 2}+\beta_{M 2}\right)+2\left(\alpha_{E 1}^{\prime}+\beta_{M 1}^{\prime}\right)\right. \\
& \left.-\alpha_{\mathrm{em}}(2 M)^{2} b_{19,0}-\frac{1}{M}\left(\delta_{L T}+\gamma_{E 1 E 1}+\gamma_{M 1 E 2}\right)+\frac{1}{(2 M)^{2}}\left(\alpha_{E 1}+\beta_{M 1}\right)\right]+\mathcal{O}\left(k^{6}\right) .
\end{aligned}
$$

We notice that the quadratic terms are fully determined by the proton electric $\left(\alpha_{E 1}\right)$ and magnetic $\left(\beta_{M 1}\right)$ dipole polarizabilities. The terms of order $\nu^{4}$ in $\bar{T}_{1}$ and of order $Q^{2} \nu^{2}$ in $\bar{T}_{2}$ are also fully determined by the electric and magnetic dispersive and quadrupole polarizabilities which are observables in RCS. The term of order $Q^{2} \nu^{2}$ in $\bar{T}_{1}$ involves in addition the slopes at $Q^{2}=0$ of the electric and magnetic GPs, as well as the RCS spin polarizabilities and the longitudinal-transverse spin polarizability $\delta_{L T}$, all of which are also observable quantities either through RCS, VCS, or using moments of spin structure functions. The only unknown in this $Q^{2} \nu^{2}$ term arises from the low-energy coefficient $b_{4,1}$. This term could in principle also be accessed from the VCS process through the LEX of the amplitude $f_{3}$, as given by Eq. (B1c), using the LEX of Eq. (9b) as

$$
b_{4,1}=\left.\frac{1}{2 M} \frac{d}{d \nu} \bar{f}_{3}(0,0, M \nu)\right|_{\nu=0},
$$

by using, e.g., a BChPT calculation for the VCS process [26]. However, it will be difficult to extract this constant empirically as it would involve higher-order GPs which have not been quantified so far. In the following, we will show, however, that a forward sum rule will allow us to fix this term.

Finally, we notice that the quartic terms of order $Q^{4}$ involve the unknown low-energy coefficients $b_{3,0}$ for $\bar{T}_{1}$ and $b_{19,0}$ for $\bar{T}_{2}$. These coefficients cannot be obtained from RCS or VCS because the corresponding tensors vanish when one or both photons are real. In this section, we will show that $b_{19,0}$ can also be determined from a forward sum rule, involving the longitudinal electroabsorption cross section on a proton. The only unknown parameter which remains then is $b_{3,0}$. Its determination will require an observable from the doubly virtual Compton process.

Having established the LEXs of the non-Born parts of the forward VVCS amplitudes $T_{1}$ and $T_{2}$, we are ready to use the analyticity in $\nu$, for fixed spacelike momentum transfer $q^{2}=-Q^{2} \leq 0$. Both amplitudes are even functions of $\nu$. We will present the relations for the nonpole parts of the amplitudes, $T_{1}^{\mathrm{np}}\left(\nu, Q^{2}\right)=T_{1}\left(\nu, Q^{2}\right)-T_{1}^{\text {pole }}\left(\nu, Q^{2}\right)$; i.e., the well-known pole amplitudes given by Eq. (17) are subtracted from the full amplitudes.

\section{Spin-independent amplitude $T_{1}$}

The dispersion relation (DR) for $T_{1}$ requires one subtraction, which we take at $\nu=0$, in order to ensure highenergy convergence,

$$
\begin{aligned}
\operatorname{Re}_{1}^{\mathrm{np}}\left(\nu, Q^{2}\right)= & T_{1}^{\mathrm{np}}\left(0, Q^{2}\right)+\frac{\nu^{2}}{2 \pi} \mathcal{P} \int_{\nu_{0}}^{\infty} d \nu^{\prime} \frac{1}{\nu^{\prime}\left(\nu^{\prime 2}-\nu^{2}\right)} \\
& \times \frac{e^{2}}{M} F_{1}\left(x^{\prime}, Q^{2}\right)
\end{aligned}
$$

with $x^{\prime} \equiv Q^{2} /\left(2 M \nu^{\prime}\right)$. Because the nonpole amplitudes are analytic functions of $\nu$, they can be expanded in a Taylor series around $\nu=0$ with a convergence radius determined by the lowest singularity, the threshold of pion production at $\nu=\nu_{0}$. Analogous to the low-energy expansion of RCS, the series in $\nu$, at fixed value of $Q^{2}$, for forward VVCS takes the following form [2],

$$
\begin{aligned}
\operatorname{Re}_{1}^{\mathrm{np}}\left(\nu, Q^{2}\right)= & T_{1}^{\mathrm{np}}\left(0, Q^{2}\right)+M_{1}^{(2)}\left(Q^{2}\right) \nu^{2} \\
& +M_{1}^{(4)}\left(Q^{2}\right) \nu^{4}+\mathcal{O}\left(\nu^{6}\right),
\end{aligned}
$$

where $M_{1}^{(2)}\left(Q^{2}\right)$ and $M_{1}^{(4)}\left(Q^{2}\right)$ can, respectively, be expressed through the second and fourth moments of the unpolarized nucleon structure function $F_{1}$ as

$$
\begin{gathered}
M_{1}^{(2)}\left(Q^{2}\right)=\frac{e^{2}(2 M)}{\pi Q^{4}} \int_{0}^{x_{0}} d x^{\prime} x^{\prime} F_{1}\left(x^{\prime}, Q^{2}\right) \\
=\frac{1}{2 \pi^{2}} \int_{\nu_{0}}^{\infty} \frac{d \nu^{\prime}}{\nu^{\prime 2}} \frac{K}{\nu^{\prime}} \sigma_{T}\left(\nu^{\prime}, Q^{2}\right), \\
M_{1}^{(4)}\left(Q^{2}\right)=\frac{e^{2}(2 M)^{3}}{\pi Q^{8}} \int_{0}^{x_{0}} d x^{\prime} x^{\prime 3} F_{1}\left(x^{\prime}, Q^{2}\right) \\
=\frac{1}{2 \pi^{2}} \int_{\nu_{0}}^{\infty} \frac{d \nu^{\prime}}{\nu^{\prime 4}} \frac{K}{\nu^{\prime}} \sigma_{T}\left(\nu^{\prime}, Q^{2}\right) .
\end{gathered}
$$

Furthermore, in the second equalities of Eqs. (26) and (27), we introduced the transverse electro-absorption cross section $\left(\sigma_{T}\right)$ on a nucleon through

$$
K \sigma_{T}\left(\nu^{\prime}, Q^{2}\right)=\frac{e^{2} \pi}{M} F_{1}\left(x^{\prime}, Q^{2}\right),
$$

where $K$ is a conveniently defined virtual photon flux factor; e.g., in the definition by Hand [27], it is given by $K=\nu^{\prime}\left(1-x^{\prime}\right)$.

To obtain the low-energy expansion of the nonpole part $T_{1}^{\mathrm{np}}$ entering Eq. (24), we also need to account for the difference between the Born and pole parts, which can be easily read off Eq. (16) as 


$$
\begin{aligned}
T_{1}^{\mathrm{Born}} & \left(\nu, Q^{2}\right)-T_{1}^{\mathrm{pole}}\left(\nu, Q^{2}\right) \\
= & -\frac{\alpha_{\mathrm{em}}}{M} F_{D}^{2} \\
= & -\frac{\alpha_{\mathrm{em}}}{M}+\frac{\alpha_{\mathrm{em}}}{3 M}\left\langle r_{1}^{2}\right\rangle Q^{2}-\frac{\alpha_{\mathrm{em}}}{M}\left(\frac{1}{36}\left\langle r_{1}^{2}\right\rangle^{2}+F_{D}^{\prime \prime}(0)\right) Q^{4} \\
& +\mathcal{O}\left(Q^{6}\right),
\end{aligned}
$$

where $\left\langle r_{1}^{2}\right\rangle$ is the squared Dirac radius of the proton and where the $Q^{4}$ term involves the curvature of the Dirac form factor at $Q^{2}=0$, defined as

$$
\left.F_{D}^{\prime \prime}(0) \equiv \frac{d^{2} F_{D}\left(Q^{2}\right)}{d\left(Q^{2}\right)^{2}}\right|_{Q^{2}=0} .
$$

As the difference between the Born and pole term contributions to $T_{1}$ is independent of $\nu$, it can be fully absorbed in the subtraction function. The non-Born part of the subtraction function $\bar{T}_{1}\left(0, Q^{2}\right)$ can be read off Eq. (21) as

$$
\begin{aligned}
\bar{T}_{1}\left(0, Q^{2}\right)= & \beta_{M 1} Q^{2}+\left(\frac{1}{6} \beta_{M 2}+2 \beta_{M 1}^{\prime}+\alpha_{\mathrm{em}} b_{3,0}\right. \\
& \left.+\frac{1}{(2 M)^{2}} \beta_{M 1}\right) Q^{4}+\mathcal{O}\left(Q^{6}\right) .
\end{aligned}
$$

Apart from the well-known fact that the expansion of $\bar{T}_{1}\left(0, Q^{2}\right)$ in powers of $Q^{2}$ starts from the term $\beta_{M 1} Q^{2}$, this relation constrains the next term in the expansion, proportional to $Q^{4}$ :

$$
\begin{aligned}
\left.\frac{1}{2} \frac{d^{2} \bar{T}_{1}\left(0, Q^{2}\right)}{d\left(Q^{2}\right)^{2}}\right|_{Q^{2}=0} \equiv & \frac{1}{2} \bar{T}_{1}^{\prime \prime}(0) \\
= & \frac{1}{6} \beta_{M 2}+2 \beta_{M 1}^{\prime}+\alpha_{\mathrm{em}} b_{3,0} \\
& +\frac{1}{(2 M)^{2}} \beta_{M 1} .
\end{aligned}
$$

Combining Eqs. (29) and (31), the subtraction function $T_{1}^{\mathrm{np}}\left(0, Q^{2}\right)$ entering the DRs of Eq. (24), including terms up to order $\mathcal{O}\left(Q^{4}\right)$, is given by

$$
\begin{aligned}
T_{1}^{\mathrm{np}}\left(0, Q^{2}\right)= & -\frac{\alpha_{\mathrm{em}}}{M}+\left[\beta_{M}+\frac{\alpha_{\mathrm{em}}}{3 M}\left\langle r_{1}^{2}\right\rangle\right] Q^{2} \\
& +\left[\frac{1}{6} \beta_{M 2}+2 \beta_{M 1}^{\prime}+\alpha_{\mathrm{em}} b_{3,0}+\frac{1}{(2 M)^{2}} \beta_{M 1}\right. \\
& \left.-\frac{\alpha_{\mathrm{em}}}{M}\left(\frac{1}{36}\left\langle r_{1}^{2}\right\rangle^{2}+F_{D}^{\prime \prime}(0)\right)\right] Q^{4}+\mathcal{O}\left(Q^{6}\right) .
\end{aligned}
$$

In order to completely fix the term of $\mathcal{O}\left(Q^{4}\right)$ in the subtraction function, one needs to determine the lowenergy coefficient $b_{3,0}$ :

$$
b_{3,0}=\frac{1}{\alpha_{\mathrm{em}}}\left\{\frac{1}{2} \bar{T}_{1}^{\prime \prime}(0)-\frac{1}{6} \beta_{M 2}-2 \beta_{M 1}^{\prime}-\frac{1}{(2 M)^{2}} \beta_{M 1}\right\} \text {. }
$$

Its determination requires a measurement of the doubly virtual Compton process with a spacelike initial and timelike final photon.

The $\nu$-dependent terms in the expansion of Eq. (25) can all be determined from sum rules in terms of electroabsorption cross sections on a nucleon, as given, e.g., by Eqs. (26) and (27). For $Q^{2}=0$, one can use the LEX of Eq. (21) to obtain the Baldin sum rule [7] and a higherorder generalization thereof as

$$
\begin{gathered}
\alpha_{E 1}+\beta_{M 1}=M_{1}^{(2)}(0)=\frac{1}{2 \pi^{2}} \int_{\nu_{0}}^{\infty} d \nu^{\prime} \frac{\sigma_{T}\left(\nu^{\prime}\right)}{\nu^{\prime 2}}, \\
\alpha_{E 1, \nu}+\beta_{M 1, \nu}+\frac{1}{12}\left(\alpha_{E 2}+\beta_{M 2}\right) \\
=M_{1}^{(4)}(0)=\frac{1}{2 \pi^{2}} \int_{\nu_{0}}^{\infty} d \nu^{\prime} \frac{\sigma_{T}\left(\nu^{\prime}\right)}{\nu^{\prime 4}},
\end{gathered}
$$

where $\sigma_{T}\left(\nu^{\prime}\right)$ is the total photoabsorption cross section on a proton.

We can next write down a new generalized Baldin sum rule for the term proportional to $Q^{2} \nu^{2}$ in the LEX of Eq. (21):

$$
\begin{aligned}
\left.\frac{d M_{1}^{(2)}\left(Q^{2}\right)}{d Q^{2}}\right|_{Q^{2}=0} \equiv & M_{1}^{(2) \prime}(0) \\
= & \beta_{M 1, \nu}+\frac{1}{12}\left(4 \beta_{M 2}+\alpha_{E 2}\right)+2\left(\alpha_{E 1}^{\prime}+\beta_{M 1}^{\prime}\right) \\
& -2 \alpha_{\mathrm{em}}(2 M)^{2} b_{4,1}+\frac{1}{M}\left(-\delta_{L T}+\gamma_{M 1 M 1}\right. \\
& \left.-\gamma_{E 1 E 1}-\gamma_{M 1 E 2}+\gamma_{E 1 M 2}\right) \\
& +\frac{1}{(2 M)^{2}}\left(\alpha_{E 1}+\beta_{M 1}\right)
\end{aligned}
$$

The structure function moment $M_{1}^{(2)}\left(Q^{2}\right)$ is an observable and has been measured at Jefferson Laboratory (JLab) [28]. If one could determine the low-energy coefficient $b_{4,1}$ from the VCS process using Eq. (23), the sum rule of Eq. (37) provides an exact nonperturbative relation which relates observables in RCS, VCS, and VVCS. A direct determination of $b_{4,1}$, however, involves higher-order GPs, which may be quite complicated to extract from experiment. In practice, one can use the measured value on the lhs of the sum rule of Eq. (37) in order to determine the low-energy coefficient $b_{4,1}$ as

$$
\begin{aligned}
b_{4,1}= & \frac{1}{\alpha_{\mathrm{em}} 8 M^{2}}\left\{-M_{1}^{(2) \prime}(0)+\beta_{M 1, \nu}+\frac{1}{12}\left(4 \beta_{M 2}+\alpha_{E 2}\right)\right. \\
& +2\left(\alpha_{E 1}^{\prime}+\beta_{M 1}^{\prime}\right)+\frac{1}{M}\left(-\delta_{L T}+\gamma_{M 1 M 1}-\gamma_{E 1 E 1}\right. \\
& \left.\left.-\gamma_{M 1 E 2}+\gamma_{E 1 M 2}\right)+\frac{1}{(2 M)^{2}}\left(\alpha_{E 1}+\beta_{M 1}\right)\right\}
\end{aligned}
$$




\section{Spin-independent amplitude $T_{2}$}

For the amplitude $T_{2}$, which is even in $\nu$, one can write down an unsubtracted DR in $\nu$ :

$$
\operatorname{Re} T_{2}^{\mathrm{np}}\left(\nu, Q^{2}\right)=\frac{1}{2 \pi} \mathcal{P} \int_{\nu_{0}}^{\infty} d \nu^{\prime} \frac{1}{\nu^{\prime 2}-\nu^{2}} e^{2} F_{2}\left(x^{\prime}, Q^{2}\right) .
$$

For the amplitude $T_{2}$, there is no difference between the Born and pole contributions, as seen from Eq. (16). The expansion of the amplitude $T_{2}^{\mathrm{np}}$ at small $k=\{\nu, Q\}$ can therefore be directly read off Eq. (22). By evaluating Eq. (39) at $\nu=0$, taking its derivative with respect to $Q^{2}$ at $Q^{2}=0$, and using the relation

$$
\left[\frac{1}{Q^{2}} F_{2}\left(x, Q^{2}\right)\right]_{Q^{2}=0}=\left[\frac{1}{Q^{2}} 2 x F_{1}\left(x, Q^{2}\right)\right]_{Q^{2}=0}=\frac{1}{\pi e^{2}} \sigma_{T},
$$

one recovers from the $Q^{2}$ term in $\bar{T}_{2}$ the Baldin sum rule of Eq. (35) and from the $Q^{2} \nu^{2}$ term in $\bar{T}_{2}$ the higher Baldin sum rule of Eq. (36).

Furthermore, Eq. (39) allows us to express $\bar{T}_{2}\left(0, Q^{2}\right)$ for general $Q^{2}$ as

$$
\bar{T}_{2}\left(0, Q^{2}\right)=Q^{2} M_{2}^{(1)}\left(Q^{2}\right)
$$

with $M_{2}^{(1)}\left(Q^{2}\right)$ the first moment of the structure function $F_{2}$,

$$
\begin{aligned}
M_{2}^{(1)}\left(Q^{2}\right)= & \frac{e^{2}(2 M)}{2 \pi Q^{4}} \int_{0}^{x_{0}} d x^{\prime} F_{2}\left(x^{\prime}, Q^{2}\right) \\
= & \frac{1}{2 \pi^{2}} \int_{\nu_{0}}^{\infty} \frac{d \nu^{\prime}}{\nu^{\prime 2}} \frac{1}{\left(1+\frac{Q^{2}}{\nu^{\prime 2}}\right)} \frac{K}{\nu^{\prime}} \\
& \times\left[\sigma_{T}\left(\nu^{\prime}, Q^{2}\right)+\sigma_{L}\left(\nu^{\prime}, Q^{2}\right)\right],
\end{aligned}
$$

where the second identity in Eq. (42) has been obtained by expressing $F_{2}$ through the sum of transverse $\left(\sigma_{T}\right)$ and longitudinal $\left(\sigma_{L}\right)$ electroabsorption cross sections on a proton as

$$
\begin{aligned}
\frac{K}{\nu^{\prime}} & {\left[\sigma_{T}\left(\nu^{\prime}, Q^{2}\right)+\sigma_{L}\left(\nu^{\prime}, Q^{2}\right)\right] } \\
& =e^{2} \pi\left(1+\frac{Q^{2}}{\nu^{\prime 2}}\right) \frac{1}{Q^{2}} F_{2}\left(x^{\prime}, Q^{2}\right) .
\end{aligned}
$$

We can then express the low-energy expansion of $\bar{T}_{2}$ including all terms up to fourth order in $k=\{\nu, Q\}$ as $^{1}$

\footnotetext{
${ }^{1}$ Note that $M_{1}^{(2)}(0)=M_{2}^{(1)}(0)$.
}

$$
\begin{aligned}
\bar{T}_{2}\left(\nu, Q^{2}\right)= & Q^{2} M_{1}^{(2)}(0)+Q^{2} \nu^{2} M_{1}^{(4)}(0) \\
& +Q^{4} M_{2}^{(1) \prime}(0)+\mathcal{O}\left(k^{6}\right)
\end{aligned}
$$

where $M_{1}^{(2)}(0)$ and $M_{1}^{(4)}(0)$ are given by Eqs. (35) and (36), respectively. The term of order $\mathcal{O}\left(Q^{4}\right)$ involves the first derivative at $Q^{2}=0$ of Eq. (42) and can be obtained through a sum-rule relation from Eq. (22) as

$$
\begin{aligned}
& \left.\frac{d M_{2}^{(1)}\left(Q^{2}\right)}{d Q^{2}}\right|_{Q^{2}=0} \\
& \equiv M_{2}^{(1) \prime}(0)=\frac{1}{6}\left(\alpha_{E 2}+\beta_{M 2}\right)+2\left(\alpha_{E 1}^{\prime}+\beta_{M 1}^{\prime}\right) \\
& \quad-\alpha_{\mathrm{em}}(2 M)^{2} b_{19,0}-\frac{1}{M}\left(\delta_{L T}+\gamma_{E 1 E 1}+\gamma_{M 1 E 2}\right) \\
& \quad+\frac{1}{(2 M)^{2}}\left(\alpha_{E 1}+\beta_{M 1}\right) .
\end{aligned}
$$

As the slope $M_{2}^{(1) \prime}(0)$ is also an observable, the knowledge of it therefore allows us to determine the low-energy coefficient $b_{19,0}$ as

$$
\begin{aligned}
b_{19,0}= & \frac{1}{\alpha_{\mathrm{em}} 4 M^{2}}\left\{-M_{2}^{(1) \prime}(0)+\frac{1}{6}\left(\alpha_{E 2}+\beta_{M 2}\right)+2\left(\alpha_{E 1}^{\prime}+\beta_{M 1}^{\prime}\right)\right. \\
& \left.-\frac{1}{M}\left(\delta_{L T}+\gamma_{E 1 E 1}+\gamma_{M 1 E 2}\right)+\frac{1}{(2 M)^{2}}\left(\alpha_{E 1}+\beta_{M 1}\right)\right\} .
\end{aligned}
$$

Let us note that it is also of interest to use the following combination of structure functions,

$$
\bar{T}_{L}\left(\nu, Q^{2}\right) \equiv-\bar{T}_{1}\left(\nu, Q^{2}\right)+\frac{\nu^{2}+Q^{2}}{Q^{2}} \bar{T}_{2}\left(\nu, Q^{2}\right),
$$

as its absorptive part can be related to the longitudinal electroabsorption cross section on a nucleon as

$$
\operatorname{Im} \bar{T}_{L}\left(\nu, Q^{2}\right)=\frac{K}{4 \pi} \sigma_{L}\left(\nu, Q^{2}\right)
$$

Its low-energy expansion, obtained by substituting $\bar{T}_{1}$ and $\bar{T}_{2}$ from Eqs. (21) and (22) into the above definition, goes as

$$
\bar{T}_{L}\left(\nu, Q^{2}\right)=Q^{2} \alpha_{E 1}+Q^{2} \nu^{2} \alpha_{L}+Q^{4} \alpha_{E}^{\prime}+O\left(k^{6}\right),
$$

with 


$$
\begin{aligned}
\alpha_{L}= & M_{2}^{(1) \prime}(0)-M_{1}^{(2) \prime}(0)+M_{1}^{(4)}(0) \\
= & \alpha_{E 1, \nu}+\frac{1}{12}\left(2 \alpha_{E 2}-\beta_{M 2}\right) \\
& +\alpha_{\mathrm{em}} 4 M^{2}\left(2 b_{4,1}-b_{19,0}\right) \\
& -\frac{1}{M}\left(\gamma_{M 1 M 1}+\gamma_{E 1 M 2}\right)
\end{aligned}
$$

where the last line has been obtained by using Eqs. (36), (37), and (45). On the other hand, we recognize that $\alpha_{L}$ is the value at $Q^{2}=0$ of the usual $\alpha_{L}\left(Q^{2}\right)$. It satisfies the sum rule in Eq. (5.36) of Hagelstein et al. [5], which at $Q^{2}=0$ corresponds with the first line in Eq. (50). Furthermore, the term of order $\mathcal{O}\left(Q^{4}\right)$ in Eq. (49) is given by

$$
\alpha_{E}^{\prime}=M_{2}^{(1) \prime}(0)-\frac{1}{2} \bar{T}_{1}^{\prime \prime}(0)=\frac{1}{6} \alpha_{E 2}+2 \alpha_{E 1}^{\prime}-\alpha_{\mathrm{em}}\left(4 M^{2} b_{19,0}+b_{3,0}\right)-\frac{1}{M}\left(\delta_{L T}+\gamma_{E 1 E 1}+\gamma_{M 1 E 2}\right)+\frac{1}{(2 M)^{2}} \alpha_{E 1} .
$$

To conclude this section, we would like to note that in Refs. $[13,14]$ a different choice of basis was used for the purpose of evaluating the Cottingham formula for the proton-neutron mass difference. The basis (denoted here by the superscript GL) used in that work is related to ours as

$$
T_{1}^{\mathrm{GL}}\left(\nu, Q^{2}\right)=-\frac{M}{\alpha_{\mathrm{em}} Q^{2}}\left[T_{1}\left(\nu, Q^{2}\right)-\frac{\nu^{2}}{Q^{2}} T_{2}\left(\nu, Q^{4}\right)\right], \quad T_{2}^{\mathrm{GL}}\left(\nu, Q^{2}\right)=\frac{M}{\alpha_{\mathrm{em}} Q^{2}} T_{2}\left(\nu, Q^{4}\right) .
$$

The LEX of the corresponding non-Born amplitudes $\bar{T}_{1}^{\mathrm{GL}}\left(\nu, Q^{2}\right)$ and $\bar{T}_{2}^{\mathrm{GL}}\left(\nu, Q^{2}\right)$ reads

$$
\begin{aligned}
\frac{\alpha_{\mathrm{em}}}{M} \bar{T}_{1}^{\mathrm{GL}}\left(\nu, Q^{2}\right)= & -\beta_{M 1}-\nu^{2}\left[\beta_{M 1, \nu}+\frac{1}{12}\left(2 \beta_{M 2}-\alpha_{E 2}\right)-\alpha_{\mathrm{em}} 4 M^{2}\left(2 b_{4,1}-b_{19,0}\right)+\frac{1}{M}\left(\gamma_{M 1 M 1}+\gamma_{E 1 M 2}\right)\right] \\
& -Q^{2}\left[\frac{1}{6} \beta_{M 2}+2 \beta_{M 1}^{\prime}+\alpha_{\mathrm{em}} b_{3,0}+\frac{1}{(2 M)^{2}} \beta_{M 1}\right]+\mathcal{O}\left(k^{4}\right) \\
\frac{\alpha_{\mathrm{em}}}{M} \bar{T}_{2}^{\mathrm{GL}}\left(\nu, Q^{2}\right)= & \left(\alpha_{E 1}+\beta_{M 1}\right)+\nu^{2}\left[\alpha_{E 1, \nu}+\beta_{M 1, \nu}+\frac{1}{12}\left(\alpha_{E 2}+\beta_{M 2}\right)\right] \\
& +Q^{2}\left[\frac{1}{6}\left(\alpha_{E 2}+\beta_{M 2}\right)+2\left(\alpha_{E 1}^{\prime}+\beta_{M 1}^{\prime}\right)-\alpha_{\mathrm{em}}(2 M)^{2} b_{19,0}\right. \\
& \left.-\frac{1}{M}\left(\delta_{L T}+\gamma_{E 1 E 1}+\gamma_{M 1 E 2}\right)+\frac{1}{(2 M)^{2}}\left(\alpha_{E 1}+\beta_{M 1}\right)\right]+\mathcal{O}\left(k^{4}\right) .
\end{aligned}
$$

To obtain the total amplitudes $T_{1}^{\mathrm{GL}}$ and $T_{2}^{\mathrm{GL}}$, one needs to add the Born terms, which read

$$
\begin{aligned}
T_{1}^{\mathrm{GL}, \mathrm{Born}} & =-\frac{F_{P}^{2}\left(q^{2}\right)}{4 M^{2}}+\frac{1}{\nu^{2}-\nu_{B}^{2}+i \varepsilon} \frac{Q^{2}}{Q^{2}+4 M^{2}}\left(G_{M}^{2}\left(q^{2}\right)-G_{E}^{2}\left(q^{2}\right)\right), \\
T_{2}^{\mathrm{GL}, \mathrm{Born}} & =-\frac{1}{\nu^{2}-\nu_{B}^{2}+i \varepsilon}\left(F_{D}^{2}\left(q^{2}\right)+\frac{Q^{2}}{4 M^{2}} F_{P}^{2}\left(q^{2}\right)\right) .
\end{aligned}
$$

The use of amplitudes $T_{1}^{\mathrm{GL}}$ and $T_{2}^{\mathrm{GL}}$ is equivalent to that of $T_{1}$ and $T_{2}$ as far as the quartic constraints derived in this work are concerned. Indeed, the $\nu^{2}$-dependent term in $T_{1}^{\mathrm{GL}}$ and the $Q^{2}$-dependent term in $T_{2}^{\mathrm{GL}}$ lead to the two new sum rules of Eqs. (37) and (45), respectively.

\section{SUM-RULE VERIFICATIONS IN BARYON CHIRAL PERTURBATION THEORY AND EMPIRICAL ESTIMATES FOR THE LOW-ENERGY COEFFICIENTS}

In this section, we verify the sum rules derived in Eqs. (37) and (45). For this purpose, we will use a covariant next-to-leading-order BChPT calculation of the non-Born part of the CS process. Furthermore, we will provide empirical estimates for the low-energy coefficients entering the sum rules.

In several previous works, we have provided next-toleading-order $\mathrm{BChPT}$ results for moments of nucleon structure functions [29], nucleon polarizabilities entering the RCS process $[30,31]$, and generalized polarizabilities entering the VCS process [26]. Such calculation is fully predictive at orders $\mathcal{O}\left(p^{3}\right)$ and $\mathcal{O}\left(p^{4} / \Delta\right)$. The $\mathcal{O}\left(p^{3}\right)$ leading-order (LO) contribution to the polarizabilities and the moments of structure functions comes from the 
TABLE I. Values of the low-energy coefficients entering the sum rule (37) for $M_{1}^{(2) \prime}(0)$, all in units of $10^{-4} \mathrm{fm}^{5}$. The first four rows are different contributions in BChPT: all columns are calculated independently in BChPT, verifying the sum rule. Errors are estimated as detailed in Ref. [31]. The last row corresponds with empirical extractions either through the experimental Bosted-Christy (BC) fit [34], dispersive (DR), or sum rule (SR) estimates as described in the text. The value of $b_{4,1}$ in the last row is the sum rule [Eq. (38)] extraction using the other values in that row as input.

\begin{tabular}{lccccccc}
\hline \hline Source & $M_{1}^{(2) \prime}(0)$ & $-\alpha_{\mathrm{em}} 8 M^{2} b_{4,1}$ & $\beta_{M 1, \nu}$ & $\left(4 \beta_{M 2}+\alpha_{E 2}\right) / 12$ & $2\left(\alpha_{E 1}^{\prime}+\beta_{M 1}^{\prime}\right)$ & $1 / M$ recoil & $1 / M^{2}$ recoil \\
\hline$\pi N$ loops & -0.74 & 2.34 & 1.78 & -1.67 & -3.53 & 0.29 & 0.06 \\
$\pi \Delta$ loops & -0.20 & 0.40 & 0.63 & -0.62 & -0.56 & -0.09 & 0.03 \\
$\Delta$ exchange & 1.00 & -1.58 & 4.72 & -1.44 & -1.41 & 0.63 & 0.08 \\
Total BChPT & $0.07 \pm 0.4$ & $1.17 \pm 0.6$ & $7.14 \pm 2.5$ & $-3.74 \pm 1.0$ & $-5.50 \pm 1.2$ & $0.83 \pm 0.3$ & $0.17 \pm 0.01$ \\
Empirical & -1.71 & 1.62 & 9.37 & -5.77 & -7.86 & 0.77 & 0.16 \\
& BC fit [34] & SR extraction & DR [35] & DR [35] & DR [2,36] & DR [2,37] & SR [5] \\
\hline \hline
\end{tabular}

pion-nucleon $(\pi N)$ loops, and the $\mathcal{O}\left(p^{4} / \Delta\right)$ NLO contribution comes from the Delta-exchange $(\Delta)$ graph and the pion-Delta $(\pi \Delta)$ loops. Here, we estimate the $\Delta(1232)$ effects in the so-called $\delta$ counting [32], with the Deltanucleon mass difference $\Delta=M_{\Delta}-M$ counted as an intermediate scale, $m_{\pi} \ll \Delta \ll M$, so that in the $\delta$ counting $m_{\pi} / \Delta \sim \Delta / M \sim \delta$.

The quoted field-theory calculations give predictions for all terms entering Eqs. (32), (37), and (45), with the exception of $b_{3,0}$ and $b_{19,0}$. The covariant BChPT thus allows us to exactly verify the sum rule (37) for $M_{1}^{(2) \prime}(0)$ by calculating all entries therein separately. The sum rule (45) for $M_{2}^{(1) \prime}(0)$ and the constraint (32) for $T_{1}^{\prime \prime}(0)$, on the other hand, can be used in order to obtain covariant BChPT predictions for the unknown coefficients $b_{3,0}$ and $b_{19,0}$. A detailed discussion of Eq. (32) is postponed to Sec. IV.

The latter two coefficients can be obtained directly from a calculation of the VVCS process in the off-forward regime. We performed such a calculation for the Deltaexchange graph, extending the CS calculation of that graph to the most general VVCS kinematics, obtaining the respective contributions to $b_{3,0}$ and $b_{19,0}$. This allows us to verify Eqs. (32) and (45), too, albeit only for the Deltaexchange graph contribution at $\mathcal{O}\left(p^{4} / \Delta\right)$.

An additional remark is in order regarding our calculation of the Delta-exchange graph. As explained in Ref. [33], the magnetic $\gamma N \Delta$ coupling $g_{M}$ is complemented by the dipole form factor, inferred from vector meson dominance considerations, and needed phenomenologically for a satisfactory description of electromagnetic nucleon-Delta transitions,

$$
g_{M} \rightarrow \frac{g_{M}}{\left[1+Q^{2} / \Lambda^{2}\right]^{2}},
$$

with the dipole mass $\Lambda^{2}=0.71 \mathrm{GeV}^{2}$. The form factor changes the slopes of the VCS GPs and the VVCS structure function moments which enter the sum rules and the analyticity constraint, specifically, the values of $\beta_{M 1}^{\prime}$,
$M_{1}^{(2) \prime}(0), M_{2}^{(1) \prime}(0)$, and $\bar{T}_{1}^{\prime \prime}(0) .{ }^{2}$ However, the sum rules and the analyticity constraint are not affected. This can be seen explicitly from the expressions for the respective Delta-exchange contributions. In general, we checked that it is possible to add an arbitrary $Q^{2}$ dependence to the couplings, e.g., by including form factors, without violating the spin-independent sum rules considered herein, or the constraint on the derivative of the subtraction function $\bar{T}_{1}^{\prime \prime}(0)$, as discussed in Sec. IV.

We show the BChPT estimates for all terms entering the sum rule (37) for $M_{1}^{(2) \prime}(0)$ in Table I and the sum rule (45) for $M_{2}^{(1) \prime}(0)$ in Table II. The values in both tables include the contribution of the dipole form factor in the Deltaexchange graph; values without those contributions can be obtained by adding $4 \beta_{M 1}^{\Delta} / \Lambda^{2}=1.57 \times 10^{-4} \mathrm{fm}^{5}$ where appropriate. Remember that $b_{4,1}$ was known from BChPT before [26], while the coefficient $b_{19,0}$ was previously unknown. For the Delta-exchange contribution, $b_{19,0}$ has been calculated directly from the off-forward VVCS process, whereas the $\pi N$ - and $\pi \Delta$-loop contributions were deduced from the sum rule and the BChPT predictions for the remaining quantities in Eq. (45).

Having verified the sum rules, we can provide empirical estimates of the low-energy coefficients. The left-hand sides of both Eqs. (37) and (45) can be estimated from the measured moments of proton structure functions. We show the empirical Bosted-Christy (BC) fit [34] for the moments $M_{1}^{(2)}$ and $M_{2}^{(1)}$ in the low- $Q^{2}$ region in Figs. 2 and 3, respectively. Their slopes at $Q^{2}=0$ are listed in Tables I and II. Furthermore, we use the dispersive estimates of Ref. [35] for the higher-order real Compton polarizabilities and of Refs. [2,36] for the GPs. We use the phenomenological MAID2007 fit [37] as input for the $\pi N$-channel contribution in the DRs. The recoil terms on the righthand sides of Eqs. (37) and (45), which are proportional to

\footnotetext{
${ }^{2}$ Note that the quantities which enter the spin-dependent sum rules considered in our previous work $[15,16]$ are not affected by these form factors.
} 
TABLE II. Values of the low-energy coefficients entering the sum rule (45) for $M_{2}^{(1) \prime}(0)$, all in units of $10^{-4} \mathrm{fm}^{5}$. The first four rows are different contributions in BChPT: the $\Delta$-exchange contributions serve as a verification of the sum rule. Errors are estimated as detailed in Ref. [31]. The last row corresponds with empirical extractions either through the experimental BC [34], DR, or SR estimates as described in the text. The value of $b_{19,0}$ in the last row is the sum rule [Eq. (46)] extraction using the other values in that row as input.

\begin{tabular}{lcccccc}
\hline \hline Source & $M_{2}^{(1) \prime}(0)$ & $-\alpha_{\mathrm{em}} 4 M^{2} b_{19,0}$ & $\left(\alpha_{E 2}+\beta_{M 2}\right) / 6$ & $2\left(\alpha_{E 1}^{\prime}+\beta_{M 1}^{\prime}\right)$ & $1 / M$ recoil & $1 / M^{2}$ recoil \\
\hline$\pi N$ loops & -1.47 & 0.95 & 0.86 & -3.53 & 0.20 & 0.06 \\
$\pi \Delta$ loops & -0.26 & 0.23 & 0.08 & -0.56 & -0.05 & 0.03 \\
$\Delta$ exchange & -1.94 & 0.01 & -0.65 & -1.41 & 0.03 & 0.08 \\
Total BChPT & $-3.68 \pm 1.1$ & $1.19 \pm 0.3$ & $0.29 \pm 0.3$ & $-5.50 \pm 1.2$ & $0.17 \pm 0.04$ & $0.17 \pm 0.01$ \\
Empirical & -6.63 & 0.14 & 0.75 & -7.86 & 0.18 & 0.16 \\
& BC fit [34] & SR extraction & DR [35] & DR [2,36] & DR [2,37] & SR [5] \\
\hline \hline
\end{tabular}

$1 / M$ and $1 / M^{2}$, depend on the lowest-order spin and scalar polarizabilities, respectively. To estimate these terms, we use the empirical values listed in Table III. Using the sum rules for $M_{1}^{(2) \prime}(0)$ and $M_{2}^{(1) \prime}(0)$, we are then able to provide empirical estimates for the low-energy coefficients $b_{4,1}$ and $b_{19,0}$, as shown in Tables I and II.

One can see from Tables I and II that there is a reasonable agreement between the BChPT values and the empirical ones for most terms entering Eqs. (37) and (45). We see differences for $M_{1}^{(2) \prime}(0)$, which is close to zero in BChPT but is negative in the empirical fit, as well as for $b_{19,0}$, which is very small in the empirical extraction. As both of these quantities yield relatively small contributions to the respective sum rules shown in Tables I and II, the differences can partly be attributed to cancellations between different terms in these relations. Figures 2 and 3 also

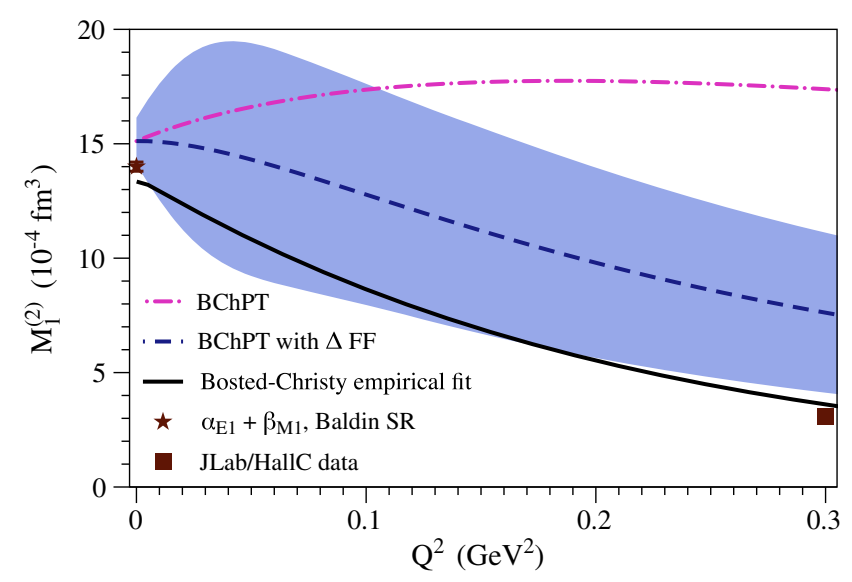

FIG. 2. $Q^{2}$ dependence of the proton structure moment $M_{1}^{(2)}$ according to the empirical BC fit (black solid curve) [34], in comparison with the $\pi N+\Delta+\pi \Delta$ BChPT calculation. For the latter, we also show the result when an additional form factor dependence is included in the $\Delta$-exchange contribution as given by Eq. (56); blue dashed (magenta dashed-dotted) curves show the results with (without) the form factor. The blue band shows the uncertainty of the BChPT result with the form factor, estimated as in Ref. [26]. At the real photon point, the observable yields the Baldin sum-rule value for $\alpha_{E 1}+\beta_{M 1}$ [5]. The data point at $Q^{2}=0.3 \mathrm{GeV}^{2}$ is from JLab/HallC [28]. demonstrate that there is qualitative agreement between the $\mathrm{BChPT}$ and the $\mathrm{BC}$ fit results for $M_{1}^{(2)}\left(Q^{2}\right)$ and $M_{2}^{(1)}\left(Q^{2}\right)$ most of the difference is just due to the different static values of $\alpha_{E 1}+\beta_{M 1}$ in the two calculations. Apart from that, the BChPT curves agree, within their (rather wide) error bands, with the $\mathrm{BC}$ fit results.

The uncertainty bands on the BChPT curves are calculated as detailed in Ref. [26] and represent a conservative estimate of corrections due to higher orders in the chiral expansion. On the other hand, one can see that the use of the form factor in the $\gamma N \Delta$ vertex is an important part of the presented result. To estimate the uncertainty due to the form factor, one notes that empirical data on electromagnetic nucleon-Delta transitions at low $Q^{2}$ allow one to extract the form factor with a precision of the order of $2 \%$; see, e.g., Ref. [38]. Varying the form factor within this range would result in changes of $M_{1}^{(2)}\left(Q^{2}\right)$ and $M_{2}^{(1)}\left(Q^{2}\right)$ at least an

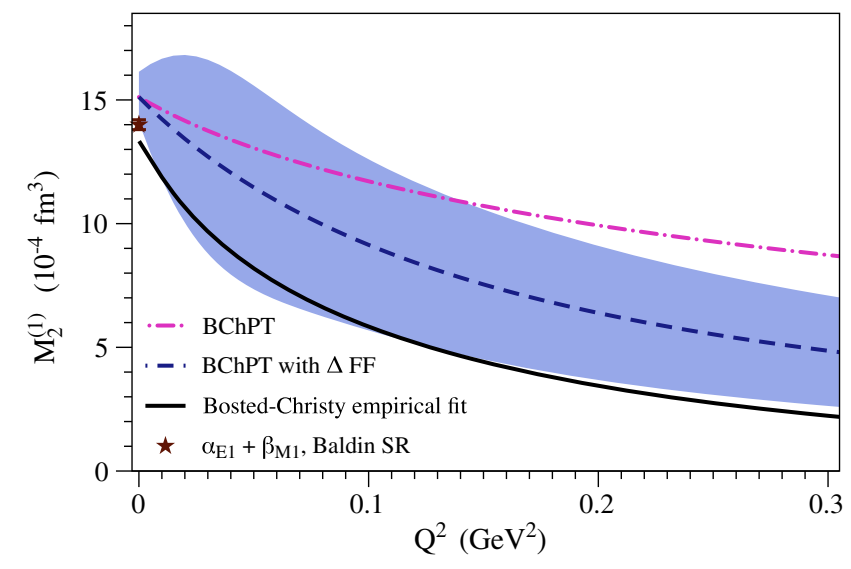

FIG. 3. $Q^{2}$ dependence of the proton structure moment $M_{2}^{(1)}$ according to the empirical BC fit (black solid curve) [34], in comparison with the $\pi N+\Delta+\pi \Delta$ BChPT calculation. For the latter, we also show the result when an additional form factor dependence is included in the $\Delta$-exchange contribution as given by Eq. (56); blue dashed (magenta dashed-dotted) curves show the results with (without) the form factor. The blue band shows the uncertainty of the BChPT result with the form factor, estimated as in Ref. [26]. At the real photon point, the observable yields the Baldin sum-rule value for $\alpha_{E 1}+\beta_{M 1}$ [5]. 
TABLE III. Empirical values for the polarizabilities used in estimating the recoil terms in Eqs. (37) and (45).

\begin{tabular}{lcc}
\hline \hline & Value & Source \\
\hline$\alpha_{E 1}+\beta_{M 1}$ & $14.0 \pm 0.2\left(10^{-4} \mathrm{fm}^{3}\right)$ & Baldin SR [5] \\
$\gamma_{E 1 E 1}$ & $-4.3\left(10^{-4} \mathrm{fm}^{4}\right)$ & DR [35] \\
$\gamma_{M 1 M 1}$ & $2.9\left(10^{-4} \mathrm{fm}^{4}\right)$ & DR [35] \\
$\gamma_{E 1 M 2}$ & $-0.1\left(10^{-4} \mathrm{fm}^{4}\right)$ & DR [35] \\
$\gamma_{M 1 E 2}$ & $2.1\left(10^{-4} \mathrm{fm}^{4}\right)$ & DR [35] \\
$\delta_{L T}$ & $1.34\left(10^{-4} \mathrm{fm}^{4}\right)$ & MAID2007 [37] \\
\hline \hline
\end{tabular}

order of magnitude smaller than the shown uncertainty bands. We thus neglect the uncertainty due to this source, expecting that any form factor that describes electromagnetic nucleon-Delta transitions reasonably well should give results close to those presented here.

The arguments concerning the uncertainty estimate also apply to the subtraction function $\bar{T}_{1}\left(0, Q^{2}\right)$; see a more detailed discussion thereof in Sec. IV.

Finally, we can also extract an empirical estimate for the longitudinal polarizability in Eq. (50). For the term $M_{1}^{(4)}(0)$, we use the empirical sum-rule evaluation of Eq. (36) yielding [39]: $M_{1}^{(4)}(0)=6.0 \times 10^{-4} \mathrm{fm}^{5}$. Using the BC fit values for $M_{1}^{(2) \prime}(0)$ and $M_{2}^{(1) \prime}(0)$, listed in Tables I and II, we then obtain an empirical estimate for $\alpha_{L}$ :

$$
\alpha_{L} \simeq 1.1 \times 10^{-4} \mathrm{fm}^{5} \text {. }
$$

This polarizability has been calculated in BChPT at NLO [29]: $\alpha_{L} \simeq 2.3 \times 10^{-4} \mathrm{fm}^{5}$. We have checked that the same value is obtained by evaluating the separate BChPT contributions in Eq. (50).

\section{LOW- $Q$ BEHAVIOR OF THE SUBTRACTION FUNCTION}

In this section, we study the $Q^{2}$ dependence of the subtraction function, $\bar{T}_{1}\left(0, Q^{2}\right)$, which is of interest for the (muonic) hydrogen Lamb shift calculations. It is the part of the TPE correction in the lepton-proton system noncalculable through the sum rules. In what follows, we will verify the analyticity constraint derived in Eq. (32) and give estimates for the low-energy coefficient $b_{3,0}$. As a result, one constrains the subtraction contribution to the Lamb shift.

The LEX given in Eq. (32) relates the second derivative of the subtraction function, $\bar{T}_{1}^{\prime \prime}(0)$, to scalar and spin polarizabilites known from RCS, the GP slope $\beta_{M 1}^{\prime}$ known from VCS, and the low-energy coefficient $b_{3,0}$. Analogously to Sec. III, we verify Eq. (32) with the Delta-exchange graph contribution at $\mathcal{O}\left(p^{4} / \Delta\right)$ in BChPT. As explained earlier, the validity of the constraint is not affected by adding a dipole form factor dependence to the magnetic coupling $g_{M}$ or, in general, by the inclusion of an arbitrary $Q^{2}$ dependence of the $\gamma N \Delta$ couplings. Once the constraint is verified, it can be used to make a prediction for $b_{3,0}$ at NLO in BChPT. As before, we rely on the results previously derived in Refs. [26,29-31]. The corresponding $\mathrm{BChPT}$ values [again, with the use of the form factor in the Delta pole, as given by Eq. (56)], as well as empirical and dispersive estimates of all quantities entering Eq. (32), are given in Table IV.

It is interesting to note that the value of $b_{3,0}$ obtained in BChPT turns out to be rather small compared to other quantities entering Eq. (32) and is driven by the Deltaexchange graph, with $\pi N$ and $\pi \Delta$ loops giving negligible contributions. The smallness of the $\pi N$ - and $\pi \Delta$-loop terms in $b_{3,0}$ could be considered accidental, given that it results from very efficient cancellations between the different terms in Eq. (32).

Let us now compare the behavior of the subtraction function in different approaches. In Fig. 4, we show $\bar{T}_{1}\left(0, Q^{2}\right) / Q^{2}$ as obtained in BChPT and heavy-baryon chiral perturbation theory (HBChPT) [42] (note that the latter calculation uses a dipole form factor (with the slope matched to the HBChPT expansion at low $Q^{2}$ ) to model the large- $Q^{2}$ behavior of the subtraction function) and an estimate from the superconvergence relation [40]. At the real photon point, $\bar{T}_{1}\left(0, Q^{2}\right) / Q^{2}$ is given by the magnetic

TABLE IV. Values of the low-energy coefficients entering the $Q^{4}$ term of the subtraction function $\bar{T}_{1}\left(0, Q^{2}\right)$, given by Eq. (32). All quantities are given in units of $10^{-4} \mathrm{fm}^{5}$. The first four rows are different contributions in BChPT: the $\Delta$-exchange contributions serve as a verification of the LEX constraint. Errors are estimated as detailed in Ref. [31]. The last row corresponds with empirical results either from DR estimates or the Particle Data Group (PDG). The value of $b_{3,0}$ in the last row is obtained from Eq. (34) by using the other values in that row as input.

\begin{tabular}{lccccc}
\hline \hline Source & $\frac{1}{2} \bar{T}_{1}^{\prime \prime}(0)$ & $\alpha_{\mathrm{em}} b_{3,0}$ & $\beta_{M 2} / 6$ & $2 \beta_{M 1}^{\prime}$ & $1 / M^{2}$ recoil \\
\hline$\pi N$ loops & -0.06 & 0.001 & -1.40 & 1.36 & -0.02 \\
$\pi \Delta$ loops & -0.10 & -0.005 & -0.44 & 0.37 & -0.02 \\
$\Delta$ exchange & -1.98 & 0.11 & -0.75 & -1.42 & 0.08 \\
Total & $-2.14 \pm 0.98$ & $0.11 \pm 0.05$ & $-2.59 \pm 0.59$ & $0.31 \pm 0.50$ & $0.04 \pm 0.01$ \\
Empirical & -0.47 & 3.96 & -4.10 & -0.36 & 0.03 \\
& estimate [40] & Eq. $(34)$ & DR [35] & DR [36,37] & PDG 2016 [41] \\
\hline \hline
\end{tabular}




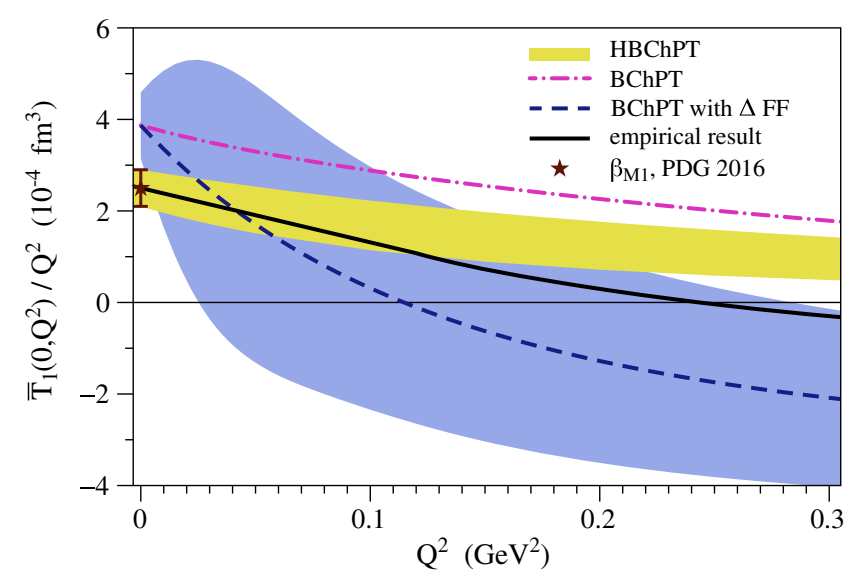

FIG. 4. The low- $Q^{2}$ behavior of the non-Born piece of the subtraction function. Shown are the HBChPT calculation [42] (dark yellow band), the BChPT calculation of this work (blue dashed and magenta dashed-dotted curves show the results with and without the form factor, respectively; the wider blue band shows the uncertainty of the BChPT result with the form factor, estimated in Ref. [26]), and the empirical superconvergence relation estimate of Ref. [40] (black solid curve). At the real photon point, the PDG 2016 value of $\beta_{M 1}=(2.5 \pm 0.4) \times$ $10^{-4} \mathrm{fm}^{3}$ [41] is shown. Note that the HBChPT curve is shifted to reproduce that value, whereas Ref. [42] uses a larger value $\beta_{M 1}=(3.15 \pm 0.50) \times 10^{-4} \mathrm{fm}^{3}$ found in the most recent HBChPT fit [43].

dipole polarizability $\beta_{M 1}$, cf. Eq. (31). The figure shows that the BChPT curve with no $\gamma N \Delta$ form factor is close to the HBChPT one; note that the static value in the latter curve was fixed to the PDG value of $\beta_{M 1}=(2.5 \pm 0.4) \times 10^{-4} \mathrm{fm}^{3}$ [41] rather than the larger value $\beta_{M 1}=(3.15 \pm 0.50) \times$ $10^{-4} \mathrm{fm}^{3}$ (which is typical of modern HBChPT [43] and BChPT [44] fits), used in Ref. [42]. The form factor on the

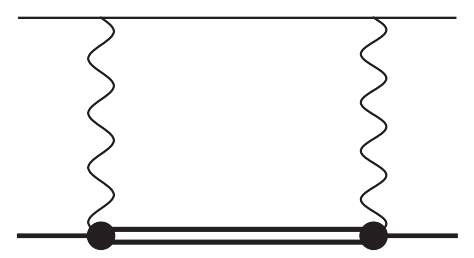

FIG. 5. Two-photon-exchange diagram with intermediate $\Delta(1232)$ excitation.

magnetic $\gamma N \Delta$ coupling increases the (negative) slope of the subtraction function at $Q^{2}=0$, as can be seen from Table IV by comparing the $\mathrm{BChPT}$ result (with form factor) with the empirical estimate. It suppresses the Delta-exchange contribution to the subtraction function at nonzero $Q^{2}$, and since the $\pi N$ - and $\pi \Delta$ - loop contributions are negative, the result with the form factor shows a zero crossing in the broad $Q^{2}$ range between 0.05 and $0.25 \mathrm{GeV}^{2}$.

Let us now turn to the contribution of the subtraction term in the TPE correction to the Lamb shift in $\mu \mathrm{H}$ and in particular to the effect of the $\Delta(1232)$ excitation, shown in Fig. 5. As seen in Fig. 4, the subtraction function changes a lot depending on the treatment of the Delta-exchange contribution. However, as argued in Ref. [45], the total contribution of the Delta exchange to the Lamb shift in $\mu \mathrm{H}$ turns out to be rather small due to cancellations between the subtraction and inelastic terms. This picture as well as the value of the total Delta-exchange contribution only very weakly depend on the parametrization of the $\gamma N \Delta$ transition. We will demonstrate it in detail below; for this purpose, we briefly recall the TPE formalism (see, e.g., Ref. [46]). The $n$th $S$-level shift in the (muonic) hydrogen spectrum due to forward TPE is related to the spinindependent forward VVCS amplitudes,

$$
\Delta E^{\mathrm{TPE}}(n S)=8 \pi e^{2} m \phi_{n}^{2} \frac{1}{i} \int_{-\infty}^{\infty} \frac{d \nu}{2 \pi} \int \frac{d \boldsymbol{q}}{(2 \pi)^{3}} \frac{\left(Q^{2}-2 \nu^{2}\right) T_{1}\left(\nu, Q^{2}\right)-\left(Q^{2}+\nu^{2}\right) T_{2}\left(\nu, Q^{2}\right)}{Q^{4}\left(Q^{4}-4 m^{2} \nu^{2}\right)},
$$

where $m$ is the lepton mass, $\phi_{n}^{2}=1 /\left(\pi n^{3} a^{3}\right)$ is the wave function at the origin, $a^{-1}=\alpha_{\mathrm{em}} m_{r}$ is the inverse Bohr radius, and $m_{r}$ is the reduced mass of the lepton-proton system. Recall also that the Lamb shift is the difference between the shifts of the $2 P$ and $2 S$ levels; the TPE contribution to the former is negligible, and the TPE contribution to the Lamb shift is thus just $-\Delta E^{\mathrm{TPE}}(2 S)$. Obviously, the polarizability effect on the hydrogen spectrum is described by the non-Born amplitudes $\bar{T}_{1}$ and $\bar{T}_{2}$. This effect can be split into the contribution of the subtraction function $\bar{T}_{1}\left(0, Q^{2}\right)$,

$$
\Delta E^{\mathrm{subtr}}(n S)=\frac{2 e^{2} m \phi_{n}^{2}}{\pi} \int_{0}^{\infty} \frac{d Q}{Q^{3}} \frac{v_{l}+2}{\left(1+v_{l}\right)^{2}} \bar{T}_{1}\left(0, Q^{2}\right)
$$

with $v_{l}=\sqrt{1+4 m^{2} / Q^{2}}$, and contributions of the inelastic structure functions (Ref. [5], Sec. 6):

$$
\begin{aligned}
\Delta E^{\text {inel }}(n S)= & -32 \alpha_{\mathrm{em}}^{2} M m \phi_{n}^{2} \int_{0}^{\infty} \frac{d Q}{Q^{5}} \int_{0}^{x_{0}} d x \frac{1}{\left(1+v_{l}\right)\left(1+\sqrt{1+x^{2} \tau^{-1}}\right)} \times\left\{\left[1+\frac{v_{l} \sqrt{1+x^{2} \tau^{-1}}}{v_{l}+\sqrt{1+x^{2} \tau^{-1}}}\right] F_{2}\left(x, Q^{2}\right)\right. \\
& \left.+\frac{2 x}{\left(1+v_{l}\right)\left(1+\sqrt{1+x^{2} \tau^{-1}}\right)}\left[2+\frac{3+v_{l} \sqrt{1+x^{2} \tau^{-1}}}{v_{l}+\sqrt{1+x^{2} \tau^{-1}}}\right] F_{1}\left(x, Q^{2}\right)\right\},
\end{aligned}
$$


TABLE V. Contribution of the $\Delta(1232)$ excitation to the $2 S$-level shift in $\mu \mathrm{H}$. All values are given in $\mu \mathrm{eV}$. For the dipole form factor (FF), we use $\Lambda^{2}=0.71 \mathrm{GeV}^{2}$. In the last row, we use the empirical parametrization [49].

\begin{tabular}{lccccc}
\hline \hline$\Delta E(2 S)$ from: & $\bar{T}_{1}\left(0, Q^{2}\right)$ Eq. $(59)$ & $F_{1}\left(x, Q^{2}\right)$ Eq. $(60)$ & $\bar{T}_{1}\left(\nu, Q^{2}\right)$ Eq. $(58)$ & $\bar{T}_{2}\left(\nu, Q^{2}\right)$ Eq. (58) & Total Eq. (58) \\
\hline$g_{M}$ (without dipole FF) & 13.19 & -4.31 & 8.88 & -7.38 & 1.50 \\
$g_{M}$ (with dipole FF) & 8.01 & -1.99 & 6.02 & -5.10 & 0.92 \\
$G_{M}^{*}, G_{E}^{*}, G_{C}^{*}$ (Jones-Scadron FFs) & 7.58 & -1.82 & 5.76 & -4.82 & 0.95 \\
\hline \hline
\end{tabular}

TABLE VI. $\Delta E^{\mathrm{TPE}}(2 S)$ contributions in different calculations, all given in $\mu \mathrm{eV}$. The last line is the sum of inelastic (inel) and subtraction (subtr) contributions.

\begin{tabular}{lrcc}
\hline \hline & & BChPT & BChPT \\
& DR/HBChPT & $($ LO $)[45]$ & $(\mathrm{LO}+\Delta)$ \\
\hline$\Delta E^{\text {inel }}(2 S)$ & $-12.7 \pm 0.5[46]$ & -5.2 & $-11.8_{-2.5}^{+2.1}$ \\
$\Delta E^{\text {subtr }}(2 S)$ & $4.2 \pm 1.0[42]$ & -3.0 & $4.6_{-2.4}^{+2.3}$ \\
$\Delta E^{\mathrm{pol}}(2 S)$ & $-8.5 \pm 1.1[51]$ & $-8.2_{-2.5}^{+1.2}$ & $-7.3_{-2.7}^{+1.5}$ \\
\hline \hline
\end{tabular}

where $\tau=Q^{2} /\left(4 M^{2}\right)$. The $\Delta(1232)$-exchange contribution to the $\bar{T}_{1}\left(0, Q^{2}\right)$ subtraction function reads [47]

$$
\begin{aligned}
\bar{T}_{1}\left(0, Q^{2}\right)= & \frac{\alpha_{\mathrm{em}} Q^{4}}{M_{\Delta} M_{+} \omega_{+}}\left[\frac{g_{M}^{2}}{Q^{2}}+\frac{g_{M} g_{E}}{M M_{+}}-\frac{g_{E}^{2} \Delta}{M^{2} M_{+}}+\frac{g_{M} g_{C}}{M M_{+}}\right. \\
& \left.+\frac{2 g_{E} g_{C}\left(M \Delta+Q^{2}\right)}{M^{2} M_{\Delta} M_{+}}-\frac{g_{C}^{2} \Delta\left(M^{2}-Q^{2}\right)}{M^{2} M_{\Delta}^{2} M_{+}}\right],
\end{aligned}
$$

with $M_{+}=M_{\Delta}+M$ and $\omega_{+}=\left(M_{\Delta}^{2}-M^{2}+Q^{2}\right) / 2 M_{\Delta}$. Here, the second row contains terms proportional to the subleading Coulomb coupling $g_{C}$.

In Table V, we show the effect of TPE with intermediate $\Delta(1232)$ excitation on the $2 S$ level in $\mu \mathrm{H}^{3}$ As mentioned above, the magnetic coupling can be multiplied by a dipole form factor in order to model a vector-meson type of dependence; the use of the form factor is specified in the table. For the prediction in the last row, the $\gamma N \Delta$ couplings were replaced by the Jones-Scadron nucleon-to-Delta transition form factors (see Ref. [47] for the details of the calculation). These transition form factors were related to nucleon form factors by the finite-momentum transfer extension of their large- $N_{c}$ limit [48]. The nucleon form factors were in turn described by an empirical parametrization [49]. As one can see from the table, the relatively large contribution of the subtraction function, $\bar{T}_{1}\left(0, Q^{2}\right)$ (second column), is largely cancelled by the contributions of the inelastic structure functions, $F_{1}$ and $F_{2}$ (third and fifth columns). The total effect of the $\Delta(1232)$ resonance on the shift of the $2 S$ state in $\mu \mathrm{H}$ is small [47] (quoting the calculation with the Jones-Scadron form factors),

\footnotetext{
${ }^{3}$ Note that the structure functions not only contain the $\Delta$ production, i.e., terms proportional to $\delta\left(x-x_{\Delta}\right)$ with $x_{\Delta}=\left[Q^{2} /\left(M_{\Delta}^{2}-M^{2}+Q^{2}\right)\right]$, but also contain terms proportional to $\delta(x)$.
}

$$
\Delta E^{\langle\Delta \text {-excit }\rangle \text { pol }}(2 S, \mu \mathrm{H})=0.95 \pm 0.95 \mu \mathrm{eV}
$$

compared to the leading effect of chiral dynamics [45],

$$
\Delta E^{\langle\mathrm{LO}\rangle \operatorname{pol}}(2 S, \mu \mathrm{H})=-8.2_{-2.5}^{+1.2} \mu \mathrm{eV} .
$$

At the same time, a calculation of the TPE with $\Delta(1232)$ excitation, employing again Jones-Scadron form factors, allows for a meaningful prediction of the contribution of the subtraction term (i.e., a prediction independent from its combination with the inelastic contribution into the polarizability contribution, cf. the discussion in Ref. [45], Sec. III) to the shift of the $2 S$ state at LO plus $\Delta$ in BChPT,

$$
\Delta E^{\langle\mathrm{LO}+\Delta\rangle \operatorname{subtr}}(2 S, \mu \mathrm{H})=4.6_{-2.4}^{+2.3} \mu \mathrm{eV},
$$

which is in good agreement with dispersive predictions $[42,46]$. Table VI shows a comparison of separate contributions to $\Delta E^{\mathrm{TPE}}(2 S)$ in different frameworks. ${ }^{4}$

To conclude this section, we note that ChPT here is an example which satisfies the sum rules. However, the hope is that the sum rules will provide a data-driven evaluation, independent of ChPT. For that, one would need to have an experimental determination of the constant $b_{3,0}$, which can become possible in future doubly virtual Compton scattering measurements.

\section{CONCLUSIONS}

The main result of this work is given by the VVCS sum rules in Eqs. (37) and (45) and the LEX constraint in Eq. (32). For the derivation, the known CS formalism, reviewed in the beginning of Sec. II, was used. At second order in energy $\left(\nu^{2}\right)$ or momentum transfer $\left(Q^{2}\right)$, the unpolarized nucleon response in the CS process is fully described in terms of electric and magnetic dipole polarizabilities. In this work, we have fully quantified the response of the double virtual CS to fourth order, including terms in $\nu^{4}, \nu^{2} Q^{2}$, and $Q^{4}$. The new forward sum rules we have derived establish relations between RCS, VCS, and VVCS observables at this order. In particular, they give access to the VVCS low-energy coefficients $b_{4,1}$ and $b_{19,0}$ through moments of the nucleon structure functions, VCS

\footnotetext{
${ }^{4} \mathrm{~A}$ different $\mathrm{HBChPT}$ prediction of the subtraction term that does not use form factors to model the high- $Q^{2}$ dependence and includes the leading and subleading $\pi N$ and $\pi \Delta$ loops, respectively, can be found in Ref. [50].
} 
GPs, and static scalar and spin polarizabilities; see Eqs. (38) and (46), respectively. From a practical point of view, this is an important result because $b_{19,0}$ does not appear in RCS or VCS experiments, and an empirical extraction of $b_{4,1}$ from VCS would be difficult due to higher-order GPs. The sum rule involving the low-energy coefficient $b_{4,1}$ was verified with the full NLO BChPT calculation, where all quantities entering Eq. (37) were calculated independently from the different CS processes. The other sum rule and the LEX constraint were verified with the $\Delta$-exchange graph contribution at $\mathcal{O}\left(p^{4} / \Delta\right)$ in $\mathrm{BChPT}$. The theoretical and empirical results for the moments of proton structure functions $M_{1}^{(2)}$ and $M_{2}^{(1)}$, cf. Figs. 2 and 3, and for most low-energy constants entering the two newly established sum rules, cf. Tables I and II, were found to be in reasonable good agreement.

The remaining unknown in the doubly virtual CS process at order $Q^{4}$ results from the low-energy coefficient $b_{3,0}$, which enters the VVCS subtraction function $\bar{T}_{1}\left(0, Q^{2}\right)$. The latter is also the main hadronic uncertainty in the estimate of the TPE correction to the muonic-hydrogen Lamb shift. Our NLO BChPT calculation yields a very small value for $b_{3,0}$. We have shown that this result originates predominantly from the $\Delta$-pole contribution. The corresponding NLO BChPT prediction of the subtraction function displays a sign change induced by the form factor dependence of the $\Delta$-exchange graph. The LO plus $\Delta \mathrm{BChPT}$ prediction for the polarizability contribution (subtraction term and inelastic term) to the $\mu \mathrm{H}$ Lamb shift is found to be in good agreement with dispersive calculations. Studying in particular the TPE with intermediate $\Delta$ excitation, we have shown that the sizeable contribution of the subtraction term is largely cancelled by the inelastic contribution, leading to a small polarizability effect of the $\Delta(1232)$ in the $\mu \mathrm{H}$ Lamb shift.

To check the smallness of the low-energy coefficient $b_{3,0}$, as predicted by our NLO BChPT calculation, we noted that there is at present no direct experimental access to the slope of the VVCS subtraction function. In order to have some empirical guidance, we compared our BChPT result with the estimate based on a superconvergence relation [40]. The latter yields a much smaller value (in absolute size) for the $Q^{4}$ term in the subtraction function $\bar{T}_{1}\left(0, Q^{2}\right)$, which then yields a significantly larger value for $b_{3,0}$. The superconvergence estimate of Ref. [40] at lower values of $Q^{2} \lesssim 1 \mathrm{GeV}^{2}$ is constrained by existing nucleon structure function data in the resonance region $(W<3 \mathrm{GeV})$ as well as by HERA data at high energies $(W>10 \mathrm{GeV})$. However, in the intermediate $W$ region $(3 \lesssim W \lesssim 10 \mathrm{GeV})$ at finite $Q^{2}$, the empirical estimate is quite uncertain because of the scarce data situation in that region. Forthcoming structure function data from the JLab $12 \mathrm{GeV}$ facility will allow us to further improve such superconvergence relation estimates for $b_{3,0}$. It may also be very worthwhile to directly access $b_{3,0}$ through a low-energy doubly virtual CS experiment. The formalism laid out in the present work provides the unpolarized hadronic tensor entering the description of such a process. We leave the study of the doubly virtual CS observables necessary to measure the low-energy coefficient $b_{3,0}$ as a topic for future work.

\section{ACKNOWLEDGMENTS}

This work was supported by the Deutsche Forschungsgemeinschaft (DFG) in part through the Collaborative Research Center [The Low-Energy Frontier of the Standard Model (SFB 1044)] and in part through the Cluster of Excellence [Precision Physics, Fundamental Interactions and Structure of Matter (PRISMA)]. The work of F.H. is supported by the Swiss National Science Foundation.

\section{APPENDIX A: RCS LIMIT}

In this Appendix, we discuss, as special case of the doubly virtual Compton process, the RCS limit, corresponding with $q^{2}=q^{2}=0$. The spin-independent part of the RCS amplitude is described by

$$
\begin{aligned}
\left.M^{\mu \nu}(\mathrm{RCS})\right|_{\text {spin indep }}= & \left(g^{\mu \nu}-\frac{q^{\prime \mu} q^{\nu}}{q \cdot q^{\prime}}\right)\left\{-q \cdot q^{\prime} B_{1}\left(0,0, q \cdot q^{\prime}, M \nu\right)-(2 M \nu)^{2} B_{2}\left(0,0, q \cdot q^{\prime}, M \nu\right)\right\} \\
& -4 q \cdot q^{\prime}\left(P^{\mu}-\frac{M \nu}{q \cdot q^{\prime}} q^{\prime \mu}\right)\left(P^{\nu}-\frac{M \nu}{q \cdot q^{\prime}} q^{\nu}\right) B_{2}\left(0,0, q \cdot q^{\prime}, M \nu\right),
\end{aligned}
$$

as the other three tensors in Eq. (4) do not contribute to the RCS limit.

Both a dispersive formulation as well as a LEX for RCS is conventionally described by an equivalent set of amplitudes $A_{i}(\nu, t)$, for $i=1, \ldots, 6$, free of kinematic singularities and constraints, see Refs. [52,53], which can be obtained as linear combinations of the $B_{i}$ amplitudes. We give here explicitly the relations between the amplitudes $B_{1}$ and $B_{2}$, which appear in Eq. (A1), and the $A_{i}$ amplitudes [36],

$$
B_{1}\left(0,0, q \cdot q^{\prime}, M \nu\right)=\frac{1}{4 \pi \alpha_{\mathrm{em}}}\left\{A_{1}(\nu, t)-A_{3}(\nu, t)-A_{6}(\nu, t)+\frac{t}{4 M^{2}} A_{3}(\nu, t)-\frac{\nu^{2}}{M^{2}} A_{4}(\nu, t)\right\}
$$




$$
B_{2}\left(0,0, q \cdot q^{\prime}, M \nu\right)=\frac{1}{4 \pi \alpha_{\mathrm{em}}} \frac{1}{2 M^{2}}\left\{A_{3}(\nu, t)+A_{6}(\nu, t)-\frac{t}{4 M^{2}} A_{4}(\nu, t)\right\},
$$

with $t=-2 q \cdot q^{\prime}$ for the RCS process.

The LEX of the non-Born parts of the amplitudes $A_{i}$ can be written as $[2,35,54]$

$\bar{A}_{i}(\nu, t)=a_{i}+a_{i, \nu} \nu^{2}+a_{i, t} t+\mathcal{O}\left(k^{4}\right), \quad i=1, \ldots, 6$,

where $k^{4}$ stands for higher-order terms either in $\nu^{4}, \nu^{2} t$, or $t^{2}$. The low-energy coefficients at zeroth order, $a_{i}$, can be expressed in terms of nucleon scalar dipole and lowestorder spin polarizabilities, whereas the low-energy coefficients at second order, $a_{i, \nu}$ and $a_{i, t}$, have been worked out in terms of quadrupole, dispersive, or higher-order spin polarizabilities $[2,35,54]$. For an example, we quote here the expressions for the combinations of the lowest-order coefficients which enter the LEXs for the amplitudes $B_{1}$ and $B_{2}$ of Eqs. (A2) and (A3),

$$
\begin{aligned}
a_{1}-a_{3}-a_{6} & =4 \pi \beta_{M 1}, \\
a_{3}+a_{6} & =-2 \pi\left(\alpha_{E 1}+\beta_{M 1}\right),
\end{aligned}
$$

in terms of the nucleon electric $\left(\alpha_{E 1}\right)$ and magnetic $\left(\beta_{M 1}\right)$ dipole polarizabilities. The detailed expressions for all coefficients $a_{i}, a_{i, t}$, and $a_{i, \nu}$ can be found in Ref. [35]. In terms of these low-energy coefficients, we can then construct the LEXs of the non-Born parts of the amplitudes $B_{1}$ and $B_{2}$ of Eqs. (A2) and (A3) in the RCS limit as

$$
\begin{gathered}
\bar{B}_{1}\left(0,0, q \cdot q^{\prime}, M \nu\right)= \\
\frac{1}{4 \pi \alpha_{\mathrm{em}}}\left\{a_{1}-a_{3}-a_{6}-2\left[a_{1, t}-a_{3, t}-a_{6, t}+\frac{a_{3}}{4 M^{2}}\right] q \cdot q^{\prime}\right. \\
\left.+\left[a_{1, \nu}-a_{3, \nu}-a_{6, \nu}-\frac{a_{4}}{M^{2}}\right] \nu^{2}\right\}+\mathcal{O}\left(k^{4}\right), \\
\bar{B}_{2}\left(0,0, q \cdot q^{\prime}, M \nu\right)=\frac{1}{4 \pi \alpha_{\mathrm{em}}} \frac{1}{2 M^{2}}\left\{a_{3}+a_{6}-2\left[a_{3, t}+a_{6, t}-\frac{a_{4}}{4 M^{2}}\right] q \cdot q^{\prime}+\left[a_{3, \nu}+a_{6, \nu}\right] \nu^{2}\right\}+\mathcal{O}\left(k^{4}\right) .
\end{gathered}
$$

By substituting the relations between the low-energy coefficients $a_{i}, a_{i, t}$, and $a_{i, \nu}$ and the polarizabilities, the RCS process then allows us to determine the coefficients in the low-energy expansion given by Eq. (9a) for the nonBorn amplitudes $\bar{B}_{1}$ and $\bar{B}_{2}$. The corresponding expressions for these coefficients are given in Eqs. (10a)-(10f). Note that the recoil terms (proportional to $1 / M$ and $1 / M^{2}$ ) in Eqs. (10a)-(10f) arise due to the transformation from the Breit frame, in which the polarizabilities such as $\beta_{M 1}, \beta_{M 1, \nu}, \beta_{M 2}, \ldots$, are defined, and the LEX of the Compton amplitude in terms of the $A_{1}, \ldots, A_{6}$.

\section{APPENDIX B: VCS LIMIT}

Another special limit of the doubly virtual Compton process is the nonforward VCS process, which corresponds with an outgoing real photon, i.e., $q^{2}=0$, and an initial spacelike virtual photon with virtuality $q^{2}=-Q^{2}<0$. The VCS process can generally be parametrized in terms of 12 independent amplitudes, $f_{i}\left(q^{2}, q \cdot q^{\prime}, q \cdot P\right)$ for $i=1, \ldots, 12$, as introduced in Ref. [24]. The nucleon spin-independent VCS process is described by three amplitudes, which are related to the doubly virtual Compton amplitudes $B_{i}$ entering Eq. (3) as

$$
\begin{aligned}
& B_{1}\left(q^{2}, 0, q \cdot q^{\prime}, q \cdot P\right)=f_{1}\left(q^{2}, q \cdot q^{\prime}, q \cdot P\right), \\
& B_{2}\left(q^{2}, 0, q \cdot q^{\prime}, q \cdot P\right)=f_{2}\left(q^{2}, q \cdot q^{\prime}, q \cdot P\right), \\
& B_{4}\left(q^{2}, 0, q \cdot q^{\prime}, q \cdot P\right)=f_{3}\left(q^{2}, q \cdot q^{\prime}, q \cdot P\right) .
\end{aligned}
$$

Note that the remaining two amplitudes $B_{3}$ and $B_{19}$ which are needed to fully specify the spin-independent doubly virtual Compton amplitude of Eq. (3) cannot be accessed in the VCS process, as the corresponding tensors decouple when the outgoing photon is real $\left(q^{\prime 2}=0\right)$.

The VCS experiments at low outgoing photon energies can also be analyzed in terms of LEXs, as proposed in Ref. [55]. For this purpose, the VCS tensor has been split in Ref. [55] into a Born part, which is defined as the nucleon intermediate state contribution using the $\gamma^{*} N N$ vertex of Eq. (7), and a non-Born part. The latter describes the response of the nucleon to the quasistatic electromagnetic field, due to the nucleon's internal structure. To obtain the lowest-order nucleon structure terms, one considers the response linear in the energy of the produced real photon. This linear response of the non-Born VCS tensor, i.e., the limit $q^{\prime} \rightarrow 0$ at arbitrary virtuality $Q^{2}$ of the initial photon, can be parametrized by six independent GPs $[55,56]$. The 
GPs can be accessed in experiment through the $e N \rightarrow e N \gamma$ process; see the reviews [1,2] for more details. At lowest order in the outgoing photon energy, there are two spinindependent GPs, denoted by $P^{(L 1, L 1) 0}$ and $P^{(M 1, M 1) 0}$, and four spin GPs, denoted by $P^{(L 1, M 2) 1}, P^{(M 1, L 2) 1}, P^{(L 1, L 1) 1}$, and $P^{(M 1, M 1) 1}$, which are all functions of $Q^{2}{ }^{5}$ In this notation, $L$ stands for the longitudinal (or electric) and $M$ stands for the magnetic nature of the transition, respectively. One usually defines the electric and magnetic GPs as

$$
\begin{aligned}
& \beta_{M 1}\left(Q^{2}\right)=-\alpha_{\mathrm{em}} \sqrt{\frac{3}{8}} P^{(M 1, M 1) 0}\left(Q^{2}\right), \\
& \alpha_{E 1}\left(Q^{2}\right)=-\alpha_{\mathrm{em}} \sqrt{\frac{3}{2}} P^{(L 1, L 1) 0}\left(Q^{2}\right),
\end{aligned}
$$

which are related to the RCS static polarizabilities as

$$
\alpha_{E 1}(0)=\alpha_{E 1}, \quad \beta_{M 1}(0)=\beta_{M 1} .
$$

The GPs can be expressed in terms of the non-Born parts $\bar{f}_{i}$ of the invariant amplitudes $f_{i}$. Using the shorthand notation

$$
\bar{f}_{i}\left(Q^{2}\right) \equiv \bar{f}_{i}\left(q^{2}=-Q^{2}, 0,0\right),
$$

the spin-independent magnetic and electric GPs can be, respectively, obtained as [56]

$$
\begin{gathered}
\beta_{M 1}\left(Q^{2}\right)=\alpha_{\mathrm{em}} \sqrt{\frac{1+\tau}{1+2 \tau}} \bar{f}_{1}\left(Q^{2}\right), \\
\alpha_{E 1}\left(Q^{2}\right)=-\alpha_{\mathrm{em}} \sqrt{\frac{1+\tau}{1+2 \tau}}\left[\bar{f}_{1}\left(Q^{2}\right)+4 M^{2}(1+\tau) \bar{f}_{2}\left(Q^{2}\right)\right. \\
\left.+Q^{2}\left(2 \bar{f}_{6}\left(Q^{2}\right)+\bar{f}_{9}\left(Q^{2}\right)-\bar{f}_{12}\left(Q^{2}\right)\right)\right] .
\end{gathered}
$$

At $Q^{2}=0$, these relations reduce to

$$
\begin{gathered}
\bar{f}_{1}(0)=\frac{1}{\alpha_{\mathrm{em}}} \beta_{M 1}, \\
\bar{f}_{2}(0)=-\frac{1}{\alpha_{\mathrm{em}}} \frac{1}{(2 M)^{2}}\left(\alpha_{E 1}+\beta_{M 1}\right) .
\end{gathered}
$$

\footnotetext{
${ }^{5}$ Equivalently, they can be considered as functions of the 3 -momentum $\overline{\mathrm{q}}$ of the virtual photon, which is conveniently defined in the c.m. system of the $\gamma^{*} N$ system, and given by $\overline{\mathrm{q}}^{2}=Q^{2}(1+\tau)$; this definition is used in Ref. [55].
}

Using the relations of Eqs. (B6a) and (B6b) as a limit of Eqs. (B1a) and (B1b), one readily verifies the expressions obtained before in Eqs. (10a) and (10d) for $b_{1,0}$ and $b_{2,0}$, respectively. We can next consider the slopes at $Q^{2}=0$ of the magnetic and electric GPs:

$$
\begin{aligned}
& \left.\beta_{M 1}^{\prime} \equiv \frac{d}{d Q^{2}} \beta_{M 1}\left(Q^{2}\right)\right|_{Q^{2}=0}, \\
& \left.\alpha_{E 1}^{\prime} \equiv \frac{d}{d Q^{2}} \alpha_{E 1}\left(Q^{2}\right)\right|_{Q^{2}=0} .
\end{aligned}
$$

By taking the derivatives at $Q^{2}=0$ of Eqs. (B5a) and (B5b), we obtain

$$
\begin{aligned}
\beta_{M 1}^{\prime}= & \alpha_{\mathrm{em}}\left[\bar{f}_{1}^{\prime}(0)-\frac{1}{8 M^{2}} \bar{f}_{1}(0)\right], \\
\alpha_{E 1}^{\prime}= & -\alpha_{\mathrm{em}}\left[\bar{f}_{1}^{\prime}(0)+4 M^{2} \bar{f}_{2}^{\prime}(0)-\frac{1}{8 M^{2}} \bar{f}_{1}(0)\right. \\
& \left.+\frac{1}{2} \bar{f}_{2}(0)+2 \bar{f}_{6}(0)+\bar{f}_{9}(0)-\bar{f}_{12}(0)\right] .
\end{aligned}
$$

The combination $2 \bar{f}_{6}(0)+\bar{f}_{9}(0)-\bar{f}_{12}(0)$ in Eq. (B8b) can be expressed in terms of spin GPs using the expressions of Ref. [56]. It was shown recently that a forward sum rule allows one to express this combination as $[15,16]$

$$
2 \bar{f}_{6}(0)+\bar{f}_{9}(0)-\bar{f}_{12}(0)=\frac{1}{\alpha_{\mathrm{em}}} \frac{1}{2 M}\left(-\delta_{L T}-\gamma_{E 1 E 1}+\gamma_{E 1 M 2}\right),
$$

in terms of the RCS spin polarizabilities $\gamma_{E 1 E 1}$ and $\gamma_{E 1 M 2}$, as well as the longitudinal-transverse spin polarizability $\delta_{L T}$ at $Q^{2}=0$, which is accessed from a moment of the nucleon spin-dependent structure functions $g_{1}$ and $g_{2}$.

We can then determine two further low-energy coefficients as

$$
\begin{aligned}
& b_{1,2 b}=-\bar{f}_{1}^{\prime}(0), \\
& b_{2,2 b}=-\bar{f}_{2}^{\prime}(0) .
\end{aligned}
$$

When using Eqs. (B8a) and (B8b), we then obtain for the coefficients $b_{1,2 b}$ and $b_{2,2 b}$ the expressions of Eqs. (11a) and (11b). 
[1] P. A. M. Guichon and M. Vanderhaeghen, Prog. Part. Nucl. Phys. 41, 125 (1998).

[2] D. Drechsel, B. Pasquini, and M. Vanderhaeghen, Phys. Rep. 378, 99 (2003).

[3] M. Schumacher, Prog. Part. Nucl. Phys. 55, 567 (2005).

[4] B. R. Holstein and S. Scherer, Annu. Rev. Nucl. Part. Sci. 64, 51 (2014).

[5] F. Hagelstein, R. Miskimen, and V. Pascalutsa, Prog. Part. Nucl. Phys. 88, 29 (2016).

[6] M. Gell-Mann, M. L. Goldberger, and W. E. Thirring, Phys. Rev. 95, 1612 (1954).

[7] A. M. Baldin, Nucl. Phys. 18, 310 (1960).

[8] S. B. Gerasimov, Yad. Fiz. 2, 598 (1965) [Sov. J. Nucl. Phys. 2, 430 (1966)].

[9] S. D. Drell and A. C. Hearn, Phys. Rev. Lett. 16, 908 (1966).

[10] H. Burkhardt and W. N. Cottingham, Ann. Phys. (Berlin) 56, 453 (1970).

[11] J. S. Schwinger, Proc. Natl. Acad. Sci. U.S.A. 72, 1 (1975); 72, 1559 (1975); Acta Phys. Austriaca Suppl. 14, 471 (1975).

[12] W. N. Cottingham, Ann. Phys. (Paris) 25, 424 (1963).

[13] J. Gasser and H. Leutwyler, Nucl. Phys. B94, 269 (1975).

[14] J. Gasser, M. Hoferichter, H. Leutwyler, and A. Rusetsky, Eur. Phys. J. C 75, 375 (2015).

[15] V. Pascalutsa and M. Vanderhaeghen, Phys. Rev. D 91, 051503 (2015).

[16] V. Lensky, V. Pascalutsa, M. Vanderhaeghen, and C. W. Kao, Phys. Rev. D 95, 074001 (2017).

[17] S. E. Kuhn, J.-P. Chen, and E. Leader, Prog. Part. Nucl. Phys. 63, 1 (2009).

[18] J. P. Chen, Int. J. Mod. Phys. E 19, 1893 (2010).

[19] P. P. Martel et al. (A2 Collaboration), Phys. Rev. Lett. 114, 112501 (2015).

[20] N. d'Hose, Eur. Phys. J. A 28, 117 (2006).

[21] P. Bourgeois et al., Phys. Rev. Lett. 97, 212001 (2006).

[22] P. Bourgeois et al., Phys. Rev. C 84, 035206 (2011).

[23] L. Correa, Ph.D. thesis, Johannes Gutenberg-Universität, Mainz and Université Blaise Pascal, Clermont-Ferrand, 2016.

[24] D. Drechsel, G. Knöchlein, A. Y. Korchin, A. Metz, and S. Scherer, Phys. Rev. C 57, 941 (1998).

[25] R. Tarrach, Nuovo Cimento A 28, 409 (1975).

[26] V. Lensky, V. Pascalutsa, and M. Vanderhaeghen, Eur. Phys. J. C 77, 119 (2017).

[27] L. N. Hand, Phys. Rev. 129, 1834 (1963).

[28] Y. Liang, M. E. Christy, R. Ent, and C. E. Keppel, Phys. Rev. C 73, 065201 (2006).

[29] V. Lensky, J. M. Alarcón, and V. Pascalutsa, Phys. Rev. C 90, 055202 (2014).

[30] V. Lensky and V. Pascalutsa, Eur. Phys. J. C 65, 195 (2010).
[31] V. Lensky, J. McGovern, and V. Pascalutsa, Eur. Phys. J. C 75, 604 (2015).

[32] V. Pascalutsa and D. R. Phillips, Phys. Rev. C 67, 055202 (2003).

[33] V. Pascalutsa and M. Vanderhaeghen, Phys. Rev. D 73, 034003 (2006).

[34] M. E. Christy and P. E. Bosted, Phys. Rev. C 81, 055213 (2010).

[35] B. R. Holstein, D. Drechsel, B. Pasquini, and M. Vanderhaeghen, Phys. Rev. C 61, 034316 (2000).

[36] B. Pasquini, M. Gorchtein, D. Drechsel, A. Metz, and M. Vanderhaeghen, Eur. Phys. J. A 11, 185 (2001).

[37] D. Drechsel, S. S. Kamalov, and L. Tiator, Eur. Phys. J. A 34, 69 (2007).

[38] K. Joo et al. (CLAS Collaboration), Phys. Rev. Lett. 88, 122001 (2002).

[39] O. Gryniuk, F. Hagelstein, and V. Pascalutsa, Phys. Rev. D 92, 074031 (2015).

[40] O. Tomalak and M. Vanderhaeghen, Eur. Phys. J. C 76, 125 (2016).

[41] C. Patrignani et al. (Particle Data Group Collaboration), Chin. Phys. C 40, 100001 (2016).

[42] M. C. Birse and J. A. McGovern, Eur. Phys. J. A 48, 120 (2012).

[43] J. A. McGovern, D. R. Phillips, and H. W. Grießhammer, Eur. Phys. J. A 49, 12 (2013).

[44] V. Lensky and J. A. McGovern, Phys. Rev. C 89, 032202 (2014).

[45] J. M. Alarcón, V. Lensky, and V. Pascalutsa, Eur. Phys. J. C 74, 2852 (2014).

[46] C. E. Carlson and M. Vanderhaeghen, Phys. Rev. A 84, 020102 (2011).

[47] F. Hagelstein, Ph.D. thesis, Johannes Gutenberg-Universität, Mainz, arXiv:1710.00874.

[48] V. Pascalutsa and M. Vanderhaeghen, Phys. Rev. D 76, 111501 (2007).

[49] R. Bradford, A. Bodek, H. S. Budd, and J. Arrington, Nucl. Phys. B, Proc. Suppl. 159, 127 (2006).

[50] C. Peset and A. Pineda, Nucl. Phys. B887, 69 (2014).

[51] A. Antognini, F. Kottmann, F. Biraben, P. Indelicato, F. Nez, and R. Pohl, Ann. Phys. (Amsterdam) 331, 127 (2013).

[52] A. I. L'vov, Yad. Fiz. 34, 1075 (1981) [Sov. J. Nucl. Phys. 34, 597 (1981)].

[53] A. I. L'vov, V. A. Petrun'kin, and M. Schumacher, Phys. Rev. C 55, 359 (1997).

[54] D. Babusci, G. Giordano, A. I. L'vov, G. Matone, and A. M. Nathan, Phys. Rev. C 58, 1013 (1998).

[55] P. A. M. Guichon, G. Q. Liu, and A. W. Thomas, Nucl. Phys. A591, 606 (1995).

[56] D. Drechsel, G. Knöchlein, A. Y. Korchin, A. Metz, and S. Scherer, Phys. Rev. C 58, 1751 (1998). 